\title{
STEREORETENTION IN THE BULK ROP OF L-LACTIDE GUIDED BY A THERMALLY STABLE ORGANOCATALYST
}

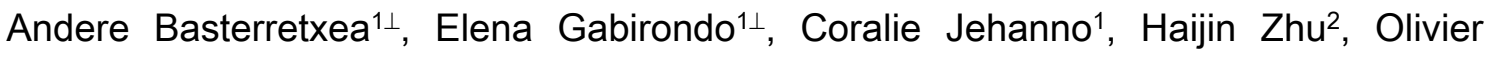 \\ Coulembier $^{3}$, David Mecerreyes ${ }^{1,4}$, Haritz Sardon ${ }^{1}$ \\ 1 POL YMAT, University of the Basque Country UPVIEHU, Paseo Manuel de Lardizabal 3, 20018, Donostia- \\ San Sebastian, Spain. \\ ${ }^{2}$ Deakin University Burwood Campus, Institute for Frontier Materials, 221 Burwood Highway, Geelong, \\ Victoria 3125, Australia \\ ${ }^{3}$ Center of Innovation and Research in Materials and Polymers (CIRMAP), Laboratory of Polymeric and \\ Composite Materials, University of Mons, Place du Parc 23, 7000 Mons, Belgium. \\ 4 IKERBASQUE Basque Foundation for Science, 48009 Bilbao, Spain \\ $\perp$ these two authors contribute equally
}

\section{Experimental Section}

\subsection{Materials}

L-Lactide (L-LA, 98\%, TCI) was recrystallized three times from toluene and dried under vacuum for two days. $\varepsilon$-Caprolactone (CLO, 99\%, Sigma Aldrich), benzyl alcohol (BnOH, 99\%, Sigma Aldrich) were dried over $\mathrm{CaH} 2$ for 48 hours prior to their distillation under reduced pressure and were stored on molecular sieves and 1-pyrenebutanol (99\%, Sigma Aldrich) were also dried under vaccum. Compounds were stored in a glove box (O2 $\leq 1 \mathrm{ppm}, \mathrm{H} 2 \mathrm{O} \leq 0.5 \mathrm{ppm})$. Methanesulfonic acid (MSA, 99\%) and 4-(dimethylamino)pyridine (DMAP, 98\%), chloroform $\left(\mathrm{CDCl}_{3}\right)$, methanol $\left(\mathrm{CH}_{3} \mathrm{OH}\right)$ and the rest of the solvents used on this work were supplied by Sigma-Aldrich and used as received.

\subsection{Characterization Techniques}

\subsubsection{Nuclear Magentic Resonance (NMR) spectroscopy}

${ }^{1} \mathrm{H}$ and ${ }^{13} \mathrm{C}$ Nuclear Magnetic Resonance (NMR) spectroscopy was recorded in a Bruker Avance DPX 300 at $300.16 \mathrm{MHz}$ and at $75.5 \mathrm{MHz}$ of resonance frequency respectively, using deuterated chloroform $\left(\mathrm{CDCl}_{3}\right)$ or as solvent at room temperature. The conditions are the following ones: (a) For $1 \mathrm{H}$ NMR spectroscopy: $10 \mathrm{mg}$ of sample; $3 \mathrm{~s}$ acquisition time; $1 \mathrm{~s}$ delay time; $8.5 \mu \mathrm{s}$ pulse; spectral width $5000 \mathrm{~Hz}$ and 32 scans. (b) For 13C NMR spectroscopy: $40 \mathrm{mg} ; 3 \mathrm{~s}$ acquisition time; $4 \mathrm{~s}$ delay time; $5.5 \mu$ s pulse; spectral width $18800 \mathrm{~Hz}$ and more than 10000 scans.

\subsubsection{Size Exclusion Chromatography (SEC)}


SEC measurements were performed at $30^{\circ} \mathrm{C}$ on an Agilent 1200 system equipped with PLgel 5 $\mu \mathrm{m}$ Guard and PLgel $5 \mu \mathrm{m}$ MIXED-C columns and a differential refractive index (RI) detector (Optilab Rex, Wyatt). THF was used as eluent at a flow rate of $1 \mathrm{ml} / \mathrm{min}$ and calibrated using narrow polystyrene standards ranging from 595 to $3.95 \cdot 10^{-6} \mathrm{~g} \mathrm{~mol}^{-1}$ (5th order universal calibration).

\subsubsection{Differential Scanning Calorimetry (DSC)}

Differential Scanning Calorimeter (DSC-Q2000, TA Instrument Inc., USA) was recorded using 6$8 \mathrm{mg}$ to calculate the glass transition and melting temperatures. First scan was carried out in all cases to eliminate the thermal history of the sample and later on the samples were analyzed between $25{ }^{\circ} \mathrm{C}$ to $160{ }^{\circ} \mathrm{C}$ at $20^{\circ} \mathrm{C} \cdot \mathrm{min}^{-1}$. The temperatures were calculated from the second heating run.

\subsubsection{Thermogravimetric analyses (TGA)}

TGA analysis were performed under nitrogen $\left(20 \mathrm{ml} \cdot \mathrm{min}^{-1}\right.$ flow rate) using a TGA Discovery (TA Instruments). The procedure is composed of a first equilibration at $100^{\circ} \mathrm{C}$ for $20 \mathrm{~min}$ and then heated at a rate of $10^{\circ} \mathrm{C} \cdot \mathrm{min}^{-1}$, in the range from $25^{\circ} \mathrm{C}$ to $800^{\circ} \mathrm{C}$.

\subsubsection{Computational details}

All calculations were carried out employing the Gaussian 16 suite of programs. Geometry optimizations were performed with the $\omega B 97 X D$ density functional and the $6-31+G(d, p)$ basis set. Frequency optimizations at the same level of theory confirmed that the optimized structures were minima (zero imaginary frequencies) or transition states (one imaginary frequency) and then used to evaluate the ZPVE and the thermal vibrational corrections at $T=298 \mathrm{~K}$. The electronic energy was refined by single-point energy calculations at the $\omega B 97 X D / 6-311++G(2 d f, 2 p)$ level of theory. The IRC method has been used to verify that the obtained transition states were connected to the desired minima.

\subsection{Methods}

\subsubsection{General procedure of the preparation of the catalyst DMAP:MSA}

In a glove box, different dual catalysts were prepared by mixing methanesulfonic acid (MSA) (1.38 $\left.10^{-4} \mathrm{~mol}, 0.013 \mathrm{~g}, 9 \mu \mathrm{l}\right)$ and 4-dimethylaminopyridine (DMAP) $\left(1.3810^{-4} \mathrm{~mol}, 0.017 \mathrm{~g}\right)$ for the mixture 1:1 while methanesulfonic acid (MSA) and $\left(2.7610^{-4} \mathrm{~mol}, 0.026 \mathrm{~g}, 18 \mu \mathrm{l}\right)$ and 4dimethylaminopyridine (DMAP) $\left(1.3810^{-4} \mathrm{~mol}, 0.017 \mathrm{~g}\right)$ were used for the preparation of the mixture $1: 2$. Afterwards, the mixtures were thermally treated at $90{ }^{\circ} \mathrm{C}$ over 30 minutes under 
stirring until complete formation of homogeneous and transparent liquid solution or salt. The good formation of those complexes was attested by $1 \mathrm{H}$ NMR spectroscopy prior their use.

1.3.2 General procedure of the Ring-Opening Polymerization of L-lactide and $\varepsilon$-caprolactone catalyzed by the DMAP:MSA mixtures

In a glove box, previously dried vials were charged with LLA $(1 \mathrm{~g})$, the DMAP:MSA catalyst (1:1, 1:2) and a stir bar. Then the initiator $(\mathrm{BnOH}$,$) was added via a 5$ or $10 \mu \mathrm{L}$ syringe. The sealed vials were immersed in a pre-heated oil bath at the desired temperature $\left(130^{\circ} \mathrm{C}-180^{\circ} \mathrm{C}\right)$. The conversion of the monomer was monitored by ${ }^{1} \mathrm{H}$ NMR spectroscopy with solution of $\mathrm{CDCl}_{3}$ until reached at least $95 \%$. The reaction was stopped by cooling down the medium at room temperature. For the purification, the crude polymer was dissolved in chloroform and precipitated in cold methanol. The resulted PLLA were filtrated and dried under vacuum at RT for $24 \mathrm{~h}$ before their characterization. The same procedure was used in the ROP of $\varepsilon$-caprolactone.

\subsubsection{Diblock copolymerization of L-lactide and $\varepsilon$-caprolactone}

In a glove box, LLA $(0.5 \mathrm{~g})$ the DMAP:MSA 1:2 and a stir bar were added to a dry vial .Then, the $\mathrm{BnOH}$ initiator was added via a 5 or $10 \mu \mathrm{L}$ syringe and the sealed vial was immersed, out of the glovebox, in pre-heated oil bath thermostatized at $130^{\circ} \mathrm{C}$. The LLA conversion of LLA was followed by ${ }^{1} \mathrm{H} \mathrm{NMR} \mathrm{(in} \mathrm{CDCl}_{3}$ ) When the conversion value was of $90 \%$ ( 8 hours), the polymerization medium was cooled down at RT and the CLO $(0.4 \mathrm{~g})$ was added in the vial by the use of a glove box. Very quickly, the vial was re-immersed at $130^{\circ} \mathrm{C}$. After $4 \mathrm{~h}$, the copolymer (PLLA-b-PCLO) was isolated by dissolving it in chloroform prior its precipitation in cold methanol. After decantation, the copolymer was filtered out and dried under vacuum. 


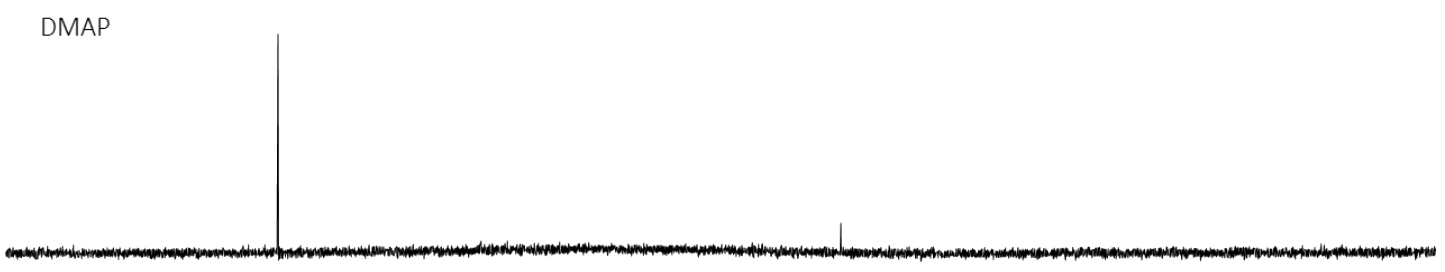

MSA:DMAP (1:1)

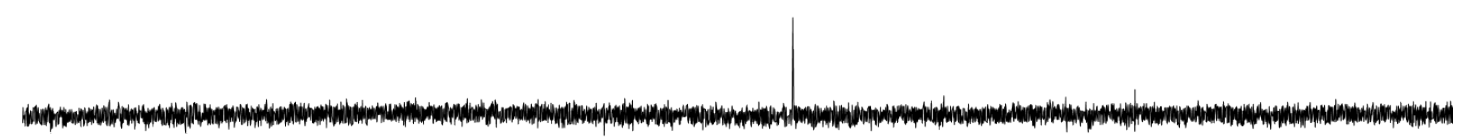
MSA:DMAP $(2: 1)$

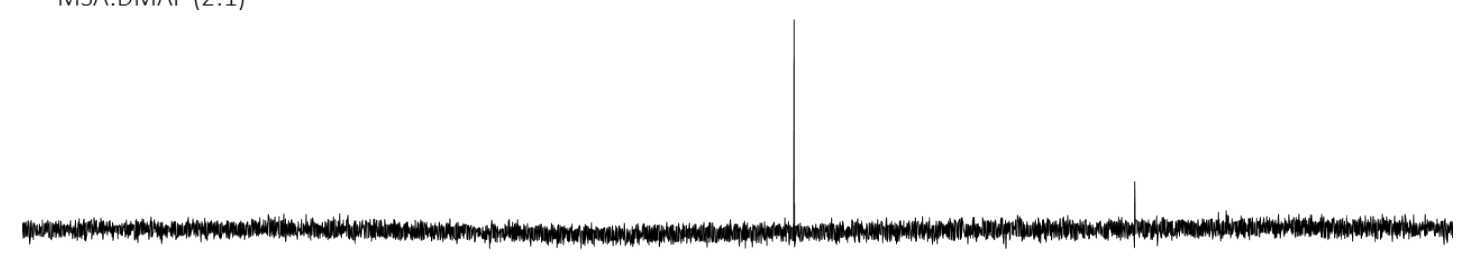

$\begin{array}{rllllllllllllllllllllllllllllllllllllllll}330 & 320 & 310 & 300 & 290 & 280 & 270 & 260 & 250 & 240 & 230 & 220 & 210 & 200 & 190 & 180 & 170 & 160 & 150 & 140 & 130 & 120 & 110 & 100 & 90 & 80 & 70 & 60 & 50 & 40 & 30 & 20\end{array}$

Figure S1. ${ }^{15}$ N NMR spectra of the catalyst DMAP, DMAP:MSA (1:1) and DMAP:MSA (1:2).

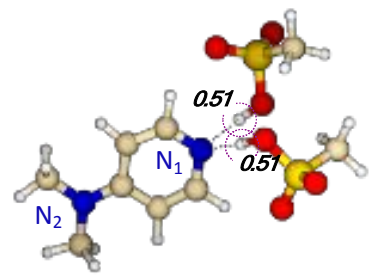

Complex (1:2) A $23.2 \mathrm{kcal} \cdot \mathrm{mol}^{-1}$

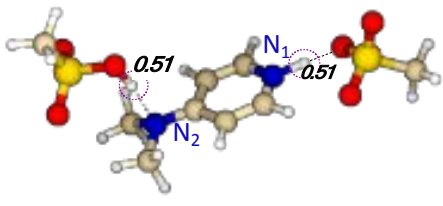

Complex (1:2) B

$30.1 \mathrm{kcal} \cdot \mathrm{mol}^{-1}$

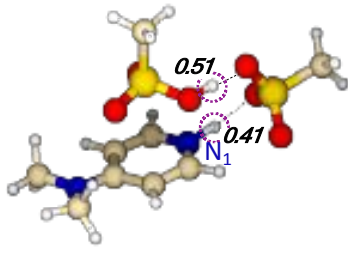

Complex (1:2) C $41.3 \mathrm{kcal} \cdot \mathrm{mol}^{-1}$

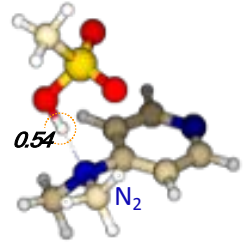

Complex (1:1) A

$12.2 \mathrm{kcal} \cdot \mathrm{mol}^{-1}$

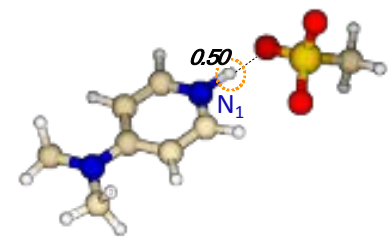

Complex (1:1) B

$19.4 \mathrm{kcal} \cdot \mathrm{mol}^{-1}$

Figure S2. Optimized structures for DMAP:MSA (1:1) (in orange) and DMAP:MSA (1:2) (in purple). Mulliken charges were extracted from DFT calculations and the resulting partial charges 
map on nitrogen atoms foreach complex demonstrate the unbalanced repartition for the less stable complexes, i.e. complex (1:1) A, complex (1:2) $A$ and complex (1:2) B. 


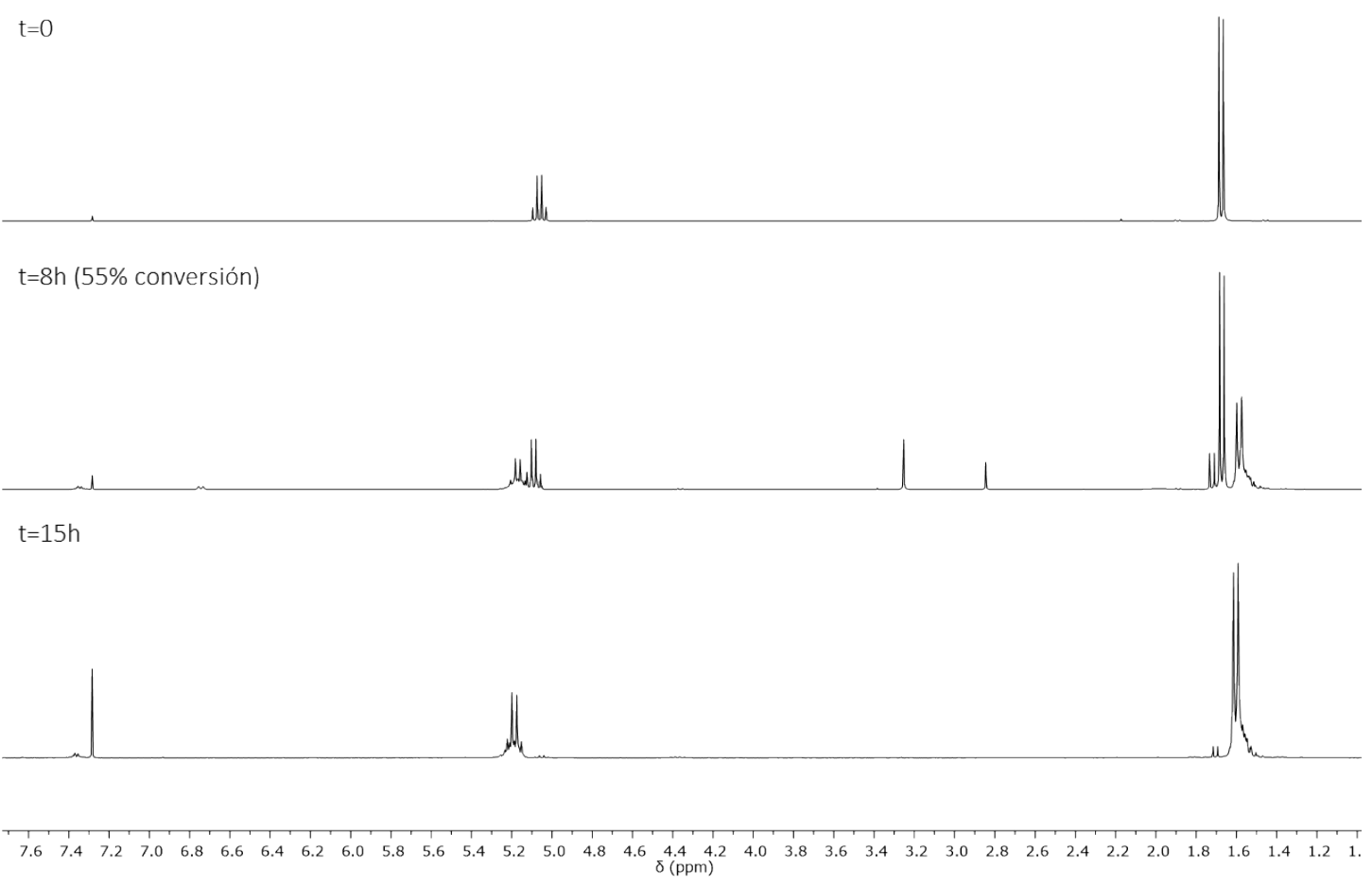

Figure S3. ${ }^{1} \mathrm{H}$ NMR spectra of the polymerization kinetics of entry 4.

a)

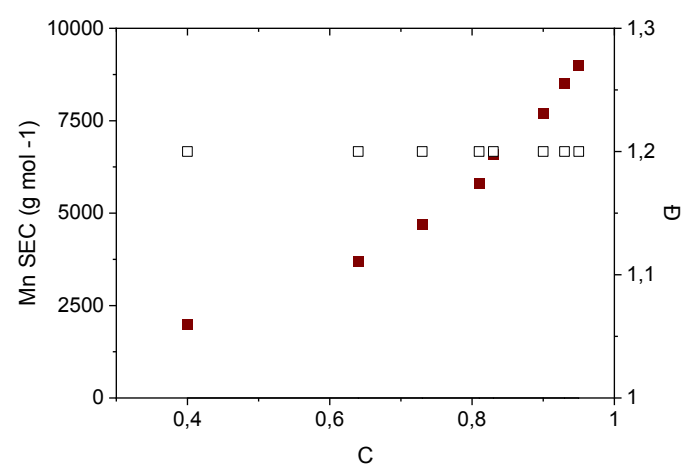

b)

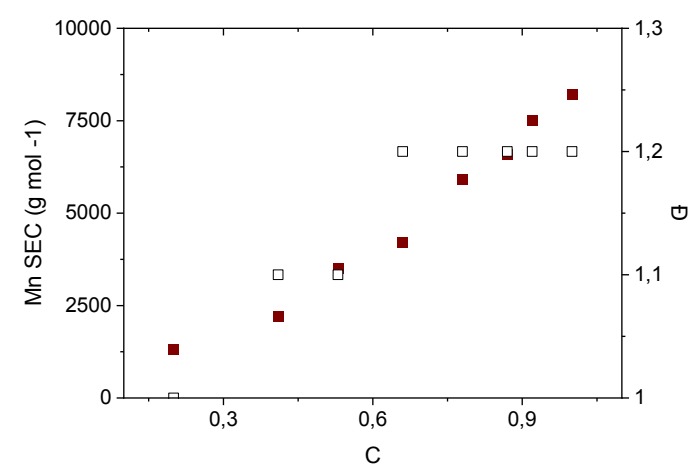

Figure S4. Mn evolution of the polymerization employing a) DMAP:MSA (1:1) and b) DMAP:MSA (1:2), entries 5 \& 6 respectively.

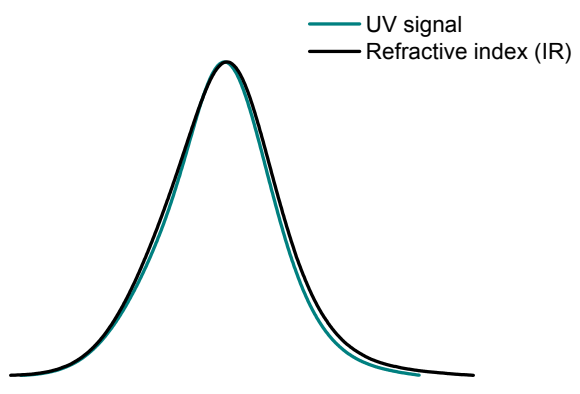


Figure S5. UV-vis and RI SEC traces for PLLA.

Entry 4

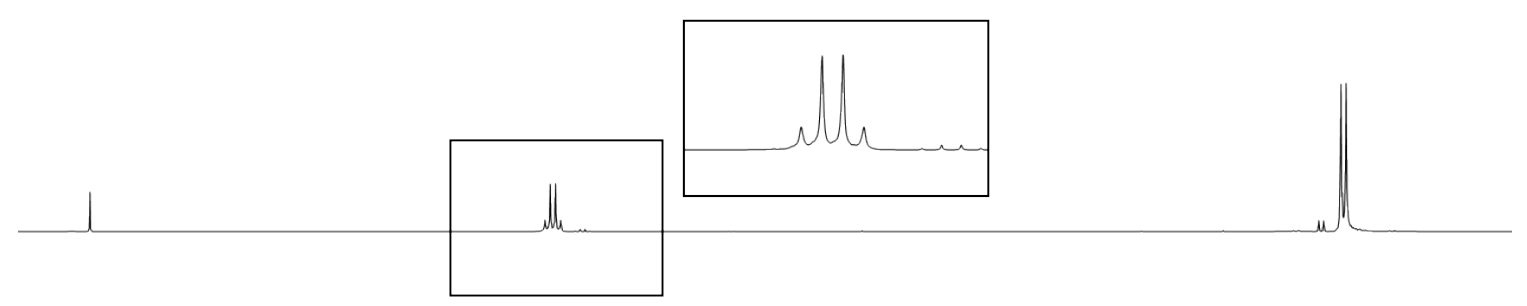

Entry 3

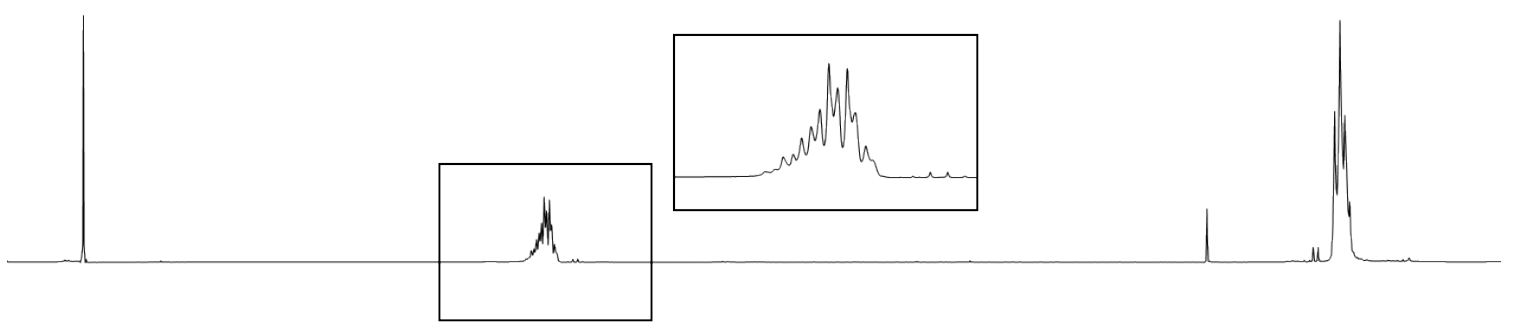

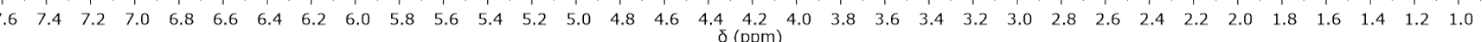

Figure S6. ${ }^{1} \mathrm{H}$ NMR of entry 4 and 3. 


\section{Entry 4}

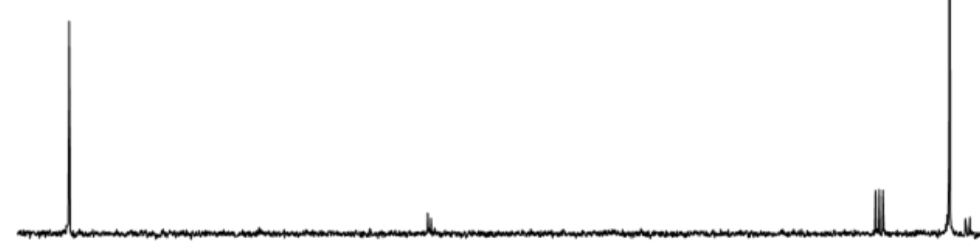

Entry 3
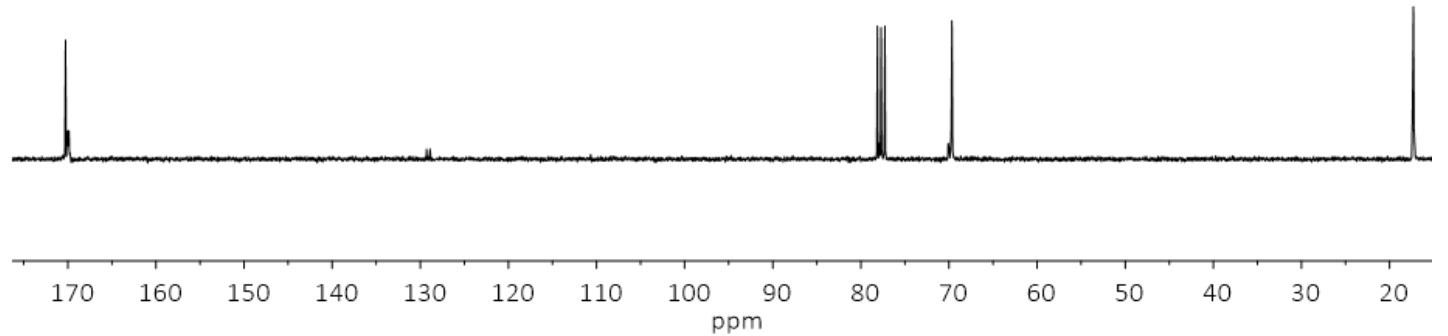

Figure S7. ${ }^{13} \mathrm{C}$ NMR of entry 4 and 3.

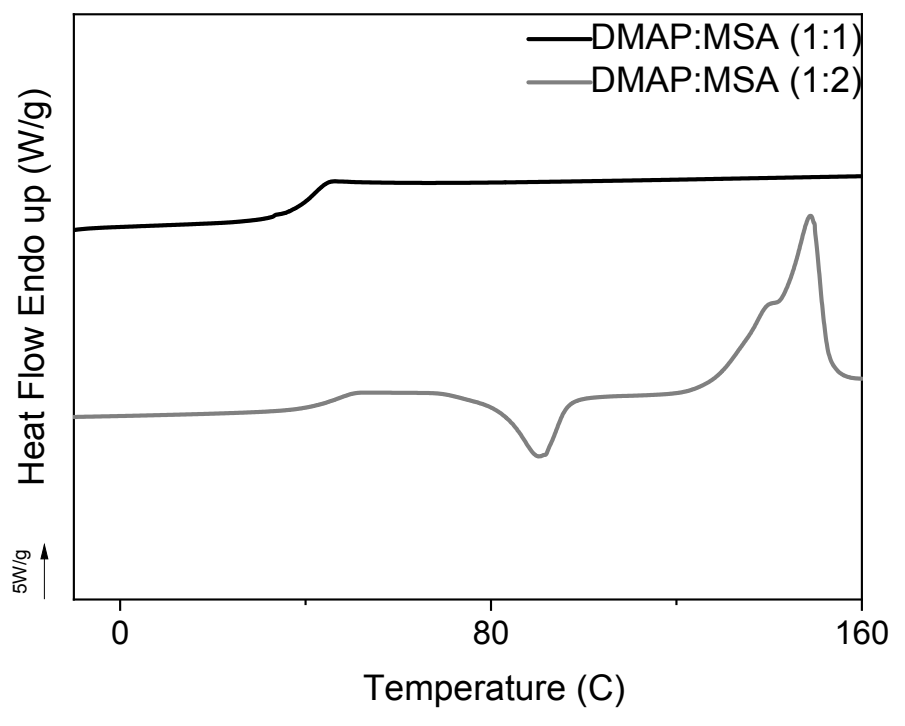


Figure S8. DSC analysis for PLA synthesized using DMAP:MSA (1:1) and DMAP:MSA (1:2).

The stereoregularity of PLA samples synthesized by DMAP:MSA (1:1) and DMAP:MSA (1:2) were determined by ${ }^{13} \mathrm{C}$ NMR taking into account the intensities of the methine region. $\mathrm{P}_{\mathrm{m}}$ is defined as the probability of mesolinkage between all the monomer units and was calculated using the methine region of ${ }^{13} \mathrm{C}$ NMR spectrum: $[\mathrm{mmm}]=\mathrm{Pm}(\mathrm{Pm}+1) / 2 ;[\mathrm{mrm}]=(1-\mathrm{Pm}) / 2 ;[\mathrm{mmr}]=\mathrm{Pm}(1-$ $\mathrm{Pm}) / 2 ;[\mathrm{rmm}]=\mathrm{Pm}(1-\mathrm{Pm}) / 2$ and $[\mathrm{rmr}]=(1-\mathrm{Pm}) 2 / 2$.

\section{Entry 4}

$\mathrm{mmm}$

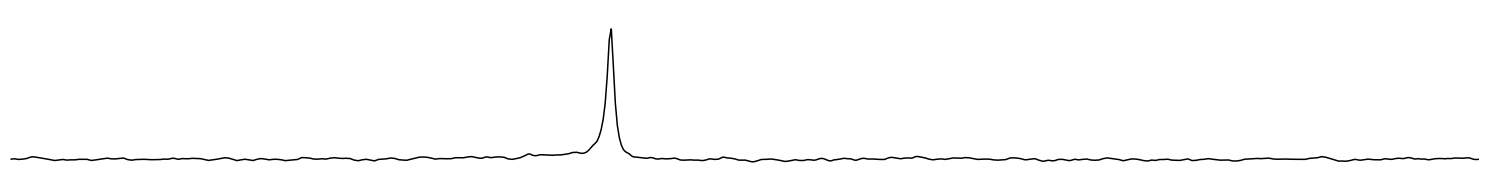

Entry 3

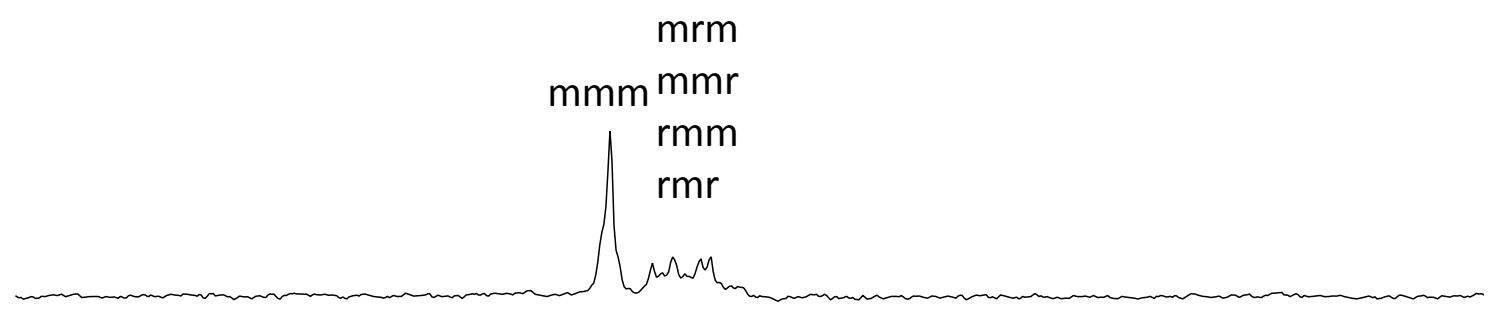

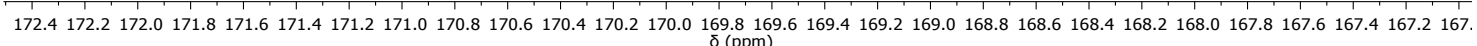

Figure S9. ${ }^{13} \mathrm{C}$ NMR analysis of PLA synthesized with DMAP:MSA (1:2) (Entry4) and DMAP:MSA (1:1) (Entry 3). 


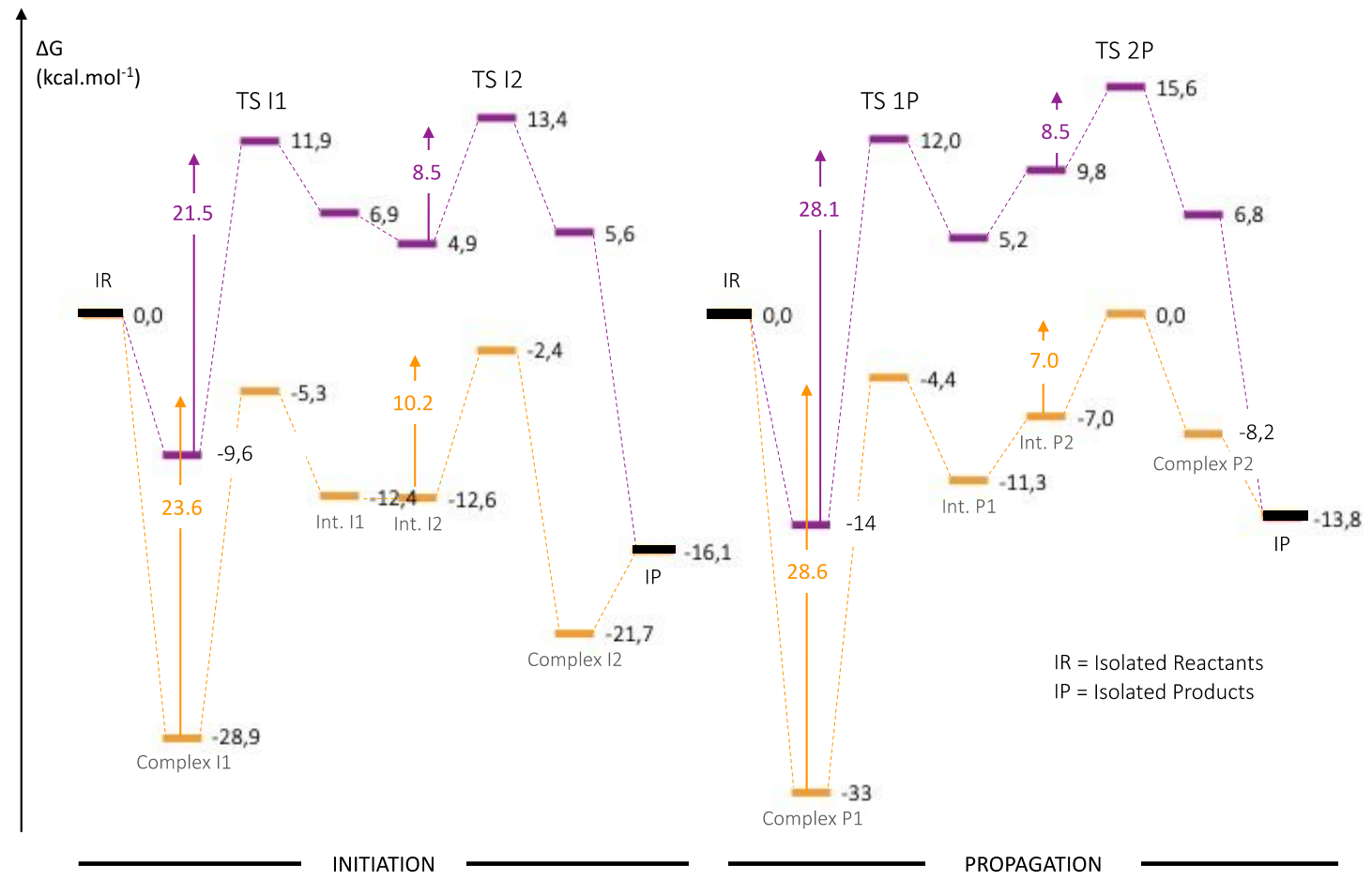

Figure S10. Energy level diagram for the initiation and the propagation of the Ring-Opening Polymerization of L-lactide with energetic stationary levels calculated at the $\omega B 97 X D / 6$ $311++G(2 d f, 2 p)$ level of theory catalyzed by DMAP:MSA (1:1) (in orange) and DMAP:MSA (1:2) (in purple).

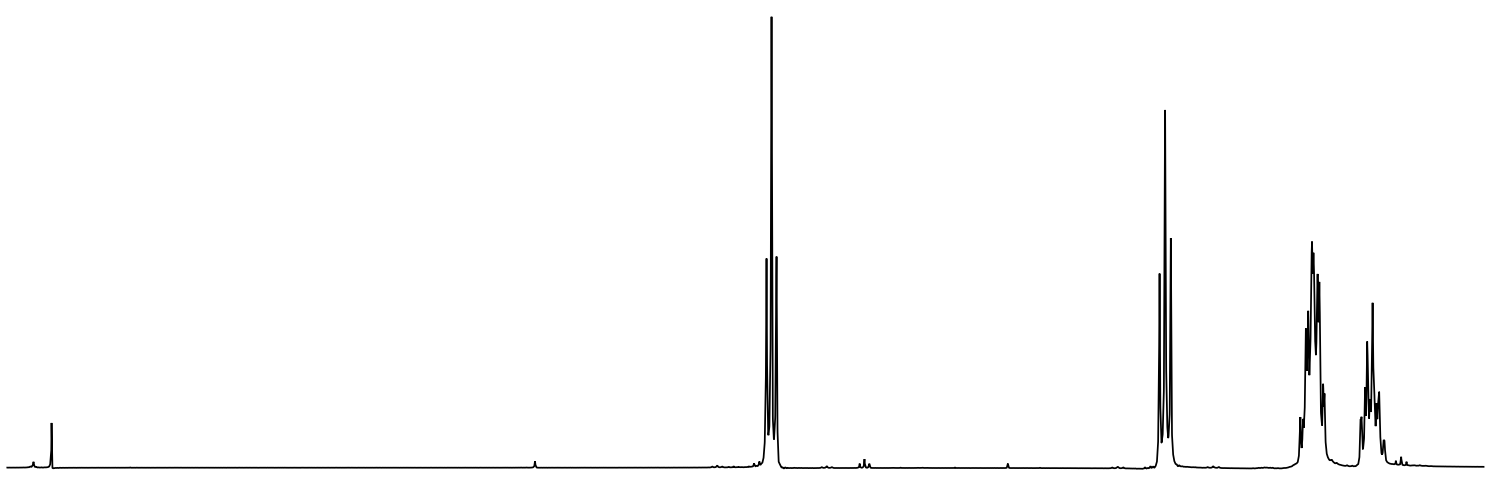

7.4 7.2 7.0 6.8 6.6 6.4 6.2 6.0 5.8 5.6 5.4 5.2 5.0 4.8 4.6 4.4 4.2 4.0 3.8 3.6 3.4 3.2 3.0 2.8 2.6 2.42 .22 .01 .81 .61 .41 .21 .0 $\delta(\mathrm{ppm})$

Figure S11. ${ }^{1}$ H NMR spectrum of PCL catalyzed by DMAP:MSA (1:2). 


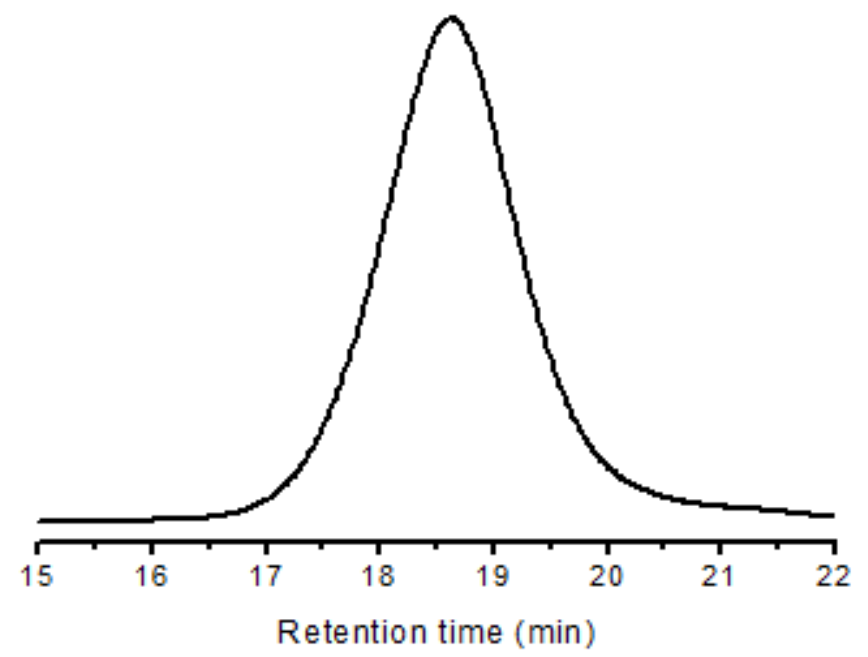

Figure S12. SEC analysis of PCL catalyzed by DMAP:MSA (1:2).

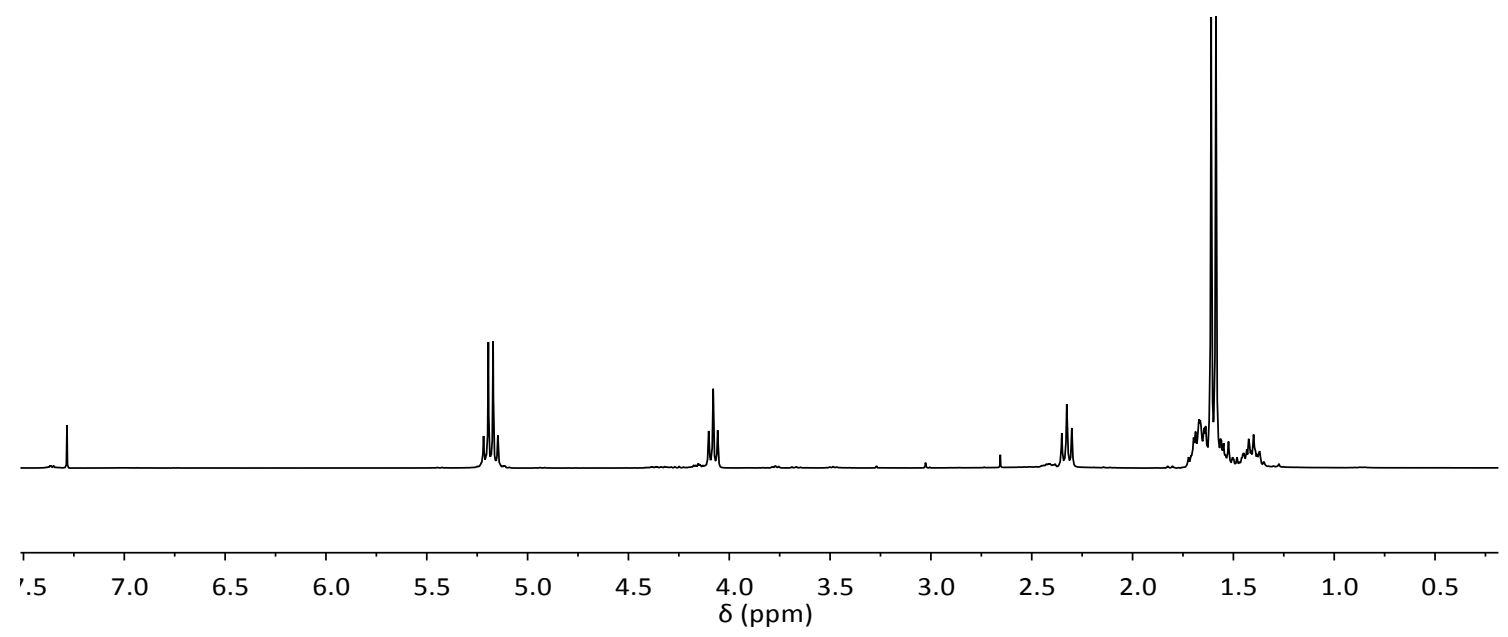

Figure S13. ${ }^{1} \mathrm{H} N M R$ spectra of PLLA-b-PCL copolymer. 


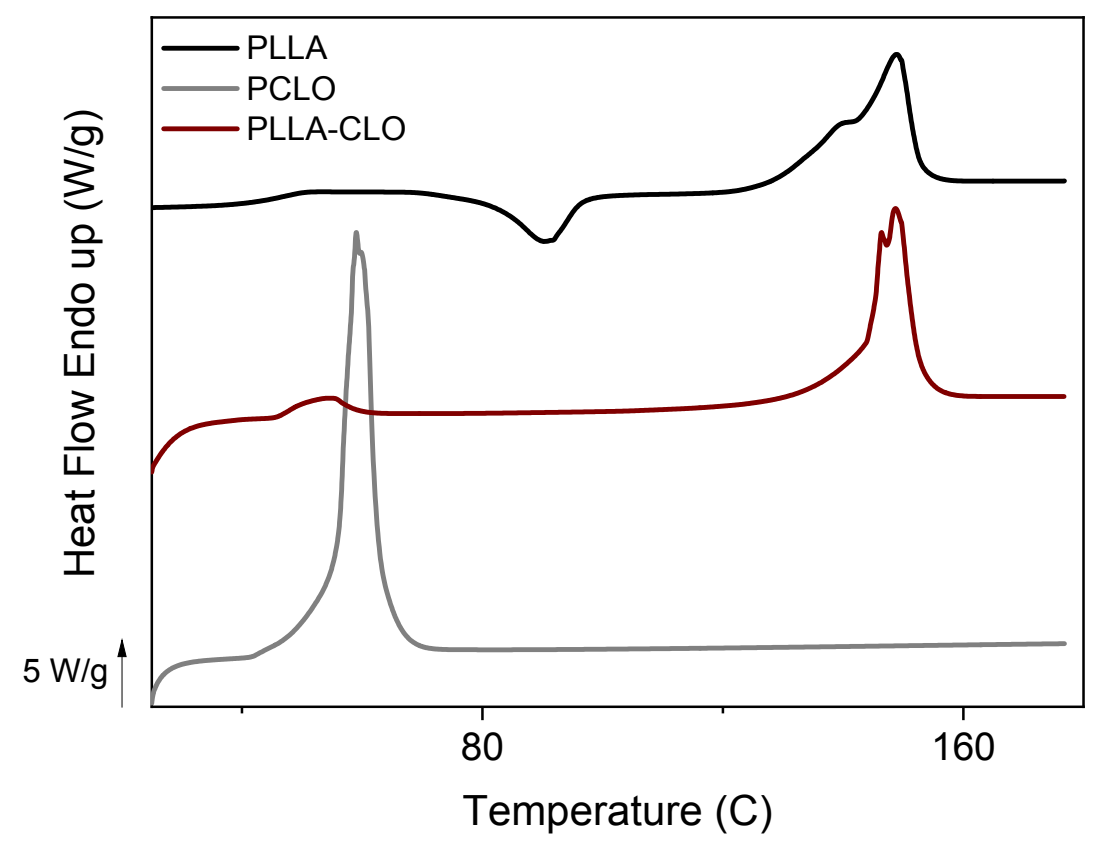

Figure S14. DSC analysis of PLLA-b-PCL copolymer. 


\section{Computational details}

All calculations were carried out employing the Gaussian 16 suite of programs. Geometry optimizations were performed with the $\omega B 97 X D$ density functional and the $6-31+G(d, p)$ basis set. Frequency optimizations at the same level of theory confirmed that the optimized structures were minima (zero imaginary frequencies) or transition states (one imaginary frequency) and then used to evaluate the ZPVE and the thermal vibrational corrections at $T=298 \mathrm{~K}$. The electronic energy was refined by single-point energy calculations at the $\omega B 97 X D / 6-311++G(2 d f, 2 p)$ level of theory. The IRC method has been used to verify that the obtained transition states were connected to the desired minima.

\section{Optimized cartesian coordinates}

For DMAP:MSA (1:1) - Initiation
Complex $/ 1$
C $\quad-4.084536 \quad-1.206064 \quad-1.072434$
$\begin{array}{llll}\mathrm{O} & -1.976227 & -0.105575 & -1.856524\end{array}$
$\begin{array}{llll}\text { C } & -1.956030 & -2.297430 & -0.764916\end{array}$
$\begin{array}{llll}\text { C } & -3.411088 & -0.167581 & -1.952887\end{array}$
$\begin{array}{llll}\text { O } & -5.266178 & -1.129130 & -0.850289\end{array}$
$\begin{array}{llll}\mathrm{H} & -1.580233 & -2.564647 & 0.223298\end{array}$
C $\quad-1.307898 \quad-0.983645 \quad-1.140964$
$\begin{array}{llll}\mathrm{O} & -0.121346 & -0.784503 & -0.923164\end{array}$
$\begin{array}{llll}\text { C } & -3.792699 & -0.369258 & -3.413079\end{array}$
H $\quad-4.877681 \quad-0.307911 \quad-3.516988$
$\begin{array}{llll}H & -3.328837 & 0.409675 & -4.022008\end{array}$
H $\quad-3.457183 \quad-1.345341 \quad-3.775324$
$\begin{array}{llll}H & -3.758828 & 0.801431 & -1.592065\end{array}$
$\begin{array}{llll}\text { C } & 3.117248 & 3.041617 & -0.864111\end{array}$
$\begin{array}{llll}\text { C } & 4.226045 & 2.286994 & -1.148288\end{array}$
N $\quad 2.112959 \quad 2.560613 \quad-0.114030$
C $\quad 4.320557 \quad 0.958552 \quad-0.646871$
H $\quad 5.004831 \quad 2.723150 \quad-1.758151$
$\begin{array}{llll}\text { C } & 2.166269 & 1.312493 & 0.388364\end{array}$
H $\quad 1.255788 \quad 3.163954 \quad 0.068641$
$\begin{array}{llll}\text { C } & 3.241055 & 0.495253 & 0.150415\end{array}$
$\begin{array}{llll}N & 5.385288 & 0.172700 & -0.915871\end{array}$
H $1.301548 \quad 1.018377 \quad 0.972693$ 


\begin{tabular}{|c|c|c|c|}
\hline $\mathrm{H}$ & 3.231648 & -0.502949 & 0.568164 \\
\hline C & 6.491027 & 0.684771 & -1.707868 \\
\hline C & 5.443984 & -1.184481 & -0.382673 \\
\hline $\mathrm{H}$ & 6.954502 & 1.557427 & -1.232684 \\
\hline$\vdash$ & 7.250243 & -0.089947 & -1.802037 \\
\hline F & 6.164668 & 0.963248 & -2.716654 \\
\hline r & 4.576276 & -1.773998 & -0.690266 \\
\hline & 6.343298 & -1.671858 & -0.756207 \\
\hline$r$ & 5.478680 & -1.182051 & 0.712075 \\
\hline C & -0.001890 & 4.081203 & 0.253130 \\
\hline$s$ & -1.153150 & 3.340293 & 0.866702 \\
\hline C & -1.818978 & 4.048079 & 1.957552 \\
\hline$c$ & -0.727558 & 1.943148 & 1.197216 \\
\hline$C$ & -2.350218 & 3.167651 & -0.454175 \\
\hline r & -2.643380 & 4.166477 & -0.778622 \\
\hline$r$ & -1.885744 & 2.609317 & -1.268422 \\
\hline$r$ & -3.210247 & 2.626703 & -0.055353 \\
\hline $\mathrm{H}$ & -1.874226 & 0.694547 & 1.082127 \\
\hline$c$ & 0.903573 & -1.539026 & 1.306638 \\
\hline r & 2.995348 & 4.054923 & -1.227750 \\
\hline r & 0.508874 & -1.303235 & 0.404168 \\
\hline C & -2.526069 & -0.025449 & 0.929545 \\
\hline C & -3.356990 & -0.149812 & 2.073642 \\
\hline $\mathrm{H}$ & -2.737360 & -0.303588 & 2.967983 \\
\hline $\mathrm{H}$ & -3.956277 & -1.053211 & 1.920140 \\
\hline C & -4.259052 & 1.061537 & 2.257643 \\
\hline $\mathrm{H}$ & -4.903286 & 0.929865 & 3.133454 \\
\hline $\mathrm{H}$ & -3.667504 & 1.971611 & 2.404904 \\
\hline $\mathrm{H}$ & -4.901921 & 1.189266 & 1.380412 \\
\hline S & 1.579209 & -2.972305 & 1.292782 \\
\hline 0 & 3.002940 & -2.833348 & 1.008764 \\
\hline O & -3.378487 & -2.251404 & -0.639465 \\
\hline C & -1.569463 & -3.369284 & -1.780035 \\
\hline $\mathrm{H}$ & -0.487901 & -3.514344 & -1.768363 \\
\hline $\mathrm{H}$ & -2.049548 & -4.307641 & -1.496328 \\
\hline $\mathrm{H}$ & -1.896867 & -3.095322 & -2.787608 \\
\hline O & 1.379875 & -3.395815 & 3.006015 \\
\hline $\mathrm{H}$ & 0.314540 & -3.443812 & 3.228786 \\
\hline $\mathrm{H}$ & 1.852072 & -4.368378 & 3.148278 \\
\hline F & 1.874067 & -2.634808 & 3.609461 \\
\hline
\end{tabular}



O
$0.787400 \quad-3.872861$
0.465489

\begin{tabular}{|c|c|c|c|}
\hline & -4.610358 & -0.943327 & -0.65976 \\
\hline & -2.534477 & -0.040874 & -1.75884 \\
\hline & -2.480109 & -2.021764 & -0.381273 \\
\hline & -3.968317 & 14791 & -1.66147 \\
\hline & -5.787991 & -0.855695 & $2472 \varepsilon$ \\
\hline & -1.981662 & -2.378509 & 0.52082 \\
\hline & -1.877086 & -0.696865 & -0.809177 \\
\hline & -0.599970 & & -0 . \\
\hline & -4.538760 & & \\
\hline & -5.621306 & -0.050766 & -3. \\
\hline & -4.100949 & 0.549985 & 5812 \\
\hline & -4.314129 & -1.186419 & -3.43306 \\
\hline & -4.2 & $1.014 \varepsilon$ & \\
\hline & 3.787460 & & \\
\hline & & & \\
\hline & 2.589370 & 5613 & -0 . \\
\hline & 4.500537 & 3260 & -0. \\
\hline & 5.703198 & 2.094801 & -1.2 \\
\hline & 2.307362 & 0.994756 & 0.1 \\
\hline & 1.863906 & & \\
\hline & 3.224026 & & \\
\hline & 5.421826 & -0.585166 & -0.9 \\
\hline & 1.31 & & 612 \\
\hline & 2.94 & -1.0 & 9755 \\
\hline & 6.719908 & -0.206446 & -1.48220 \\
\hline & & & \\
\hline & & & \\
\hline & 7.318165 & -1.1 & \\
\hline & 6.623951 & 0.302733 & \\
\hline & 4.245778 & -2.311600 & -1.320820 \\
\hline & 5.971691 & -2.595648 & -1.023655 \\
\hline & 4.881678 & -2.198003 & 0.31932 \\
\hline & 0.760740 & 4.212050 & 0.387667 \\
\hline & -0.603092 & 3.662069 & 0.627548 \\
\hline & -1.184246 & 3.969861 & 1.93349 \\
\hline
\end{tabular}




$\begin{array}{lrrr}\mathrm{O} & -0.594370 & 2.190460 & 0.305427 \\ \mathrm{C} & -1.650517 & 4.409984 & -0.611738 \\ \mathrm{H} & -1.637435 & 5.488981 & -0.455580 \\ \mathrm{H} & -1.258926 & 4.155479 & -1.596733 \\ \mathrm{H} & -2.661220 & 4.020907 & -0.482114 \\ \mathrm{H} & -1.680849 & 1.161619 & 0.484309 \\ \mathrm{O} & 0.602796 & -1.553060 & 0.987355 \\ \mathrm{H} & 3.934402 & 3.729761 & -0.738695 \\ \mathrm{H} & -0.081244 & -1.093822 & -0.184503 \\ \mathrm{O} & -2.313672 & 0.369577 & 0.609850 \\ \mathrm{C} & -2.183897 & -0.085220 & 1.979551 \\ \mathrm{H} & -1.139759 & -0.361148 & 2.155385 \\ \mathrm{H} & -2.812118 & -0.974137 & 2.062605 \\ \mathrm{C} & -2.664102 & 0.999653 & 2.923007 \\ \mathrm{H} & -2.571630 & 0.642165 & 3.953320 \\ \mathrm{H} & -2.074142 & 1.914437 & 2.819982 \\ \mathrm{H} & -3.715405 & 1.234124 & 2.734452 \\ \mathrm{~S} & 1.073334 & -2.998285 & 1.047681 \\ \mathrm{O} & 2.452140 & -3.125911 & 0.549205 \\ \mathrm{O} & -3.862302 & -1.863657 & -0.039570 \\ \mathrm{C} & -2.309251 & -3.058269 & -1.483139 \\ \mathrm{H} & -1.249702 & -3.284783 & -1.609995 \\ \mathrm{H} & -2.815958 & -3.973759 & -1.173119 \\ \mathrm{H} & -2.734044 & -2.711291 & -2.429216 \\ \mathrm{C} & 1.120473 & -3.342145 & 2.799326 \\ \mathrm{H} & 0.114582 & -3.225476 & 3.203395 \\ \mathrm{H} & 1.465587 & -4.368808 & 2.925306 \\ \mathrm{H} & 1.811262 & -2.643322 & 3.271300 \\ \mathrm{O} & 0.077545 & -3.897441 & 0.448084\end{array}$

Int. la
C $\quad-4.817406 \quad-0.815263 \quad-0.493151$
$\begin{array}{llll}\mathrm{O} & -2.832472 & 0.475176 & -1.276628\end{array}$
$\begin{array}{llll}C & -2.617604 & -1.813672 & -0.645007\end{array}$
$\begin{array}{llll}\text { C } & -4.229446 & 0.506446 & -0.994333\end{array}$
$\begin{array}{llll}\mathrm{O} & -5.995817 & -0.898782 & -0.247260\end{array}$
H $\quad-2.110252 \quad-2.491321 \quad 0.044232$
$\begin{array}{llll}\text { C } & -2.099865 & -0.382425 & -0.462250\end{array}$ 


\begin{tabular}{|c|c|c|c|}
\hline 0 & -0.803885 & -0.231784 & -0.847646 \\
\hline C & -4.937977 & 0.974454 & -2.255466 \\
\hline $\mathrm{H}$ & -6.009501 & 1.055515 & -2.067241 \\
\hline $\mathrm{H}$ & -4.549086 & 1.952743 & -2.547833 \\
\hline $\mathrm{H}$ & -4.769729 & 0.270834 & -3.075230 \\
\hline $\mathrm{H}$ & -4.407647 & 1.218792 & -0.179619 \\
\hline C & 3.893388 & 2.731126 & -0.017480 \\
\hline 0 & 4.885994 & 1.885944 & -0.432037 \\
\hline$N$ & 2.712038 & 2.258190 & 0.426863 \\
\hline 0 & 4.671096 & 0.475049 & -0.409985 \\
\hline $\mathrm{H}$ & 5.814990 & 2.315931 & -0.779823 \\
\hline C & 2.471281 & 0.926384 & 0.474790 \\
\hline $\mathrm{H}$ & 1.965739 & 2.921866 & 0.681124 \\
\hline C & 3.413156 & 0.021702 & 0.074977 \\
\hline $\mathrm{N}$ & 5.607133 & -0.392704 & -0.830178 \\
\hline $\mathrm{H}$ & 1.499014 & 0.612256 & 0.833599 \\
\hline $\mathrm{H}$ & 3.156058 & -1.032711 & 0.125052 \\
\hline C & 6.897496 & 0.088389 & -1.297838 \\
\hline C & 5.339762 & -1.832005 & -0.793226 \\
\hline $\mathrm{H}$ & 7.423286 & 0.646812 & -0.514784 \\
\hline $\mathrm{H}$ & 7.514772 & -0.763952 & -1.575766 \\
\hline $\mathrm{H}$ & 6.787987 & 0.729883 & -2.179761 \\
\hline $\mathrm{H}$ & 4.432371 & -2.084474 & -1.346997 \\
\hline $\mathrm{H}$ & 6.182778 & -2.355857 & -1.240623 \\
\hline $\mathrm{H}$ & 5.206095 & -2.184627 & 0.233681 \\
\hline O & 0.578758 & 4.066939 & 0.699906 \\
\hline$S$ & -0.690355 & 3.374602 & 0.436845 \\
\hline O & -1.934080 & 4.009374 & 0.819441 \\
\hline O & -0.505452 & 1.982037 & 1.169918 \\
\hline C & -0.747356 & 2.954497 & -1.288478 \\
\hline $\mathrm{H}$ & -0.810782 & 3.896967 & -1.834295 \\
\hline $\mathrm{H}$ & 0.159907 & 2.405945 & -1.540835 \\
\hline $\mathrm{H}$ & -1.619238 & 2.323203 & -1.468276 \\
\hline $\mathrm{H}$ & -1.267270 & 1.332856 & 1.028672 \\
\hline $\mathrm{O}$ & 0.723038 & -1.564258 & 0.815933 \\
\hline $\mathrm{H}$ & 4.003537 & 3.808493 & -0.028864 \\
\hline $\mathrm{H}$ & -0.216903 & -0.810139 & -0.288790 \\
\hline 0 & -2.305000 & 0.114233 & 0.875950 \\
\hline 0 & -2.183632 & -0.777904 & 2.007824 \\
\hline $\mathrm{H}$ & -1.195901 & -1.248572 & 1.983904 \\
\hline
\end{tabular}




$\begin{array}{lrrr}\mathrm{H} & -2.963498 & -1.540866 & 1.931933 \\ \mathrm{C} & -2.378890 & 0.051462 & 3.260188 \\ \mathrm{H} & -2.341235 & -0.603685 & 4.135149 \\ \mathrm{H} & -1.596703 & 0.807739 & 3.366308 \\ \mathrm{H} & -3.350661 & 0.552187 & 3.245969 \\ \mathrm{~S} & 1.128256 & -2.980810 & 0.483156 \\ \mathrm{O} & 2.546201 & -3.046495 & 0.072432 \\ \mathrm{O} & -4.012709 & -1.863746 & -0.276122 \\ \mathrm{C} & -2.423680 & -2.323183 & -2.060018 \\ \mathrm{H} & -1.358150 & -2.454004 & -2.252953 \\ \mathrm{H} & -2.913335 & -3.294801 & -2.152738 \\ \mathrm{H} & -2.847783 & -1.630045 & -2.791608 \\ \mathrm{C} & 1.021978 & -3.846851 & 2.045437 \\ \mathrm{H} & -0.010423 & -3.805361 & 2.395121 \\ \mathrm{H} & 1.322569 & -4.881265 & 1.875931 \\ \mathrm{H} & 1.691098 & -3.364142 & 2.758221 \\ \mathrm{O} & 0.164856 & -3.618966 & -0.425095\end{array}$

Int. Ib

$\begin{array}{lrrr}\text { C } & 4.085744 & -1.789972 & 0.109306 \\ \text { O } & 2.114241 & -0.312371 & -0.163629 \\ \text { C } & 2.359750 & -1.624759 & 1.805386 \\ \text { C } & 3.323428 & -0.783828 & -0.756269 \\ \text { O } & 5.132627 & -2.246708 & -0.277993 \\ \text { H } & 1.914569 & -2.433547 & 2.388274 \\ \text { C } & 1.426562 & -1.270870 & 0.638864 \\ \text { O } & 0.305112 & -0.695472 & 1.126732 \\ \text { C } & 4.190509 & 0.423972 & -1.075241 \\ \text { H } & 5.102965 & 0.097403 & -1.575817 \\ \text { H } & 3.638759 & 1.103086 & -1.730272 \\ \text { H } & 4.467040 & 0.949743 & -0.154719 \\ \text { H } & 3.071062 & -1.320395 & -1.676658 \\ \text { C } & -1.619048 & 1.711334 & 1.679630 \\ \mathrm{C} & -2.525706 & 0.711312 & 1.876261 \\ \text { N } & -1.813598 & 2.657184 & 0.739052 \\ \text { C } & -3.669919 & 0.614114 & 1.033536 \\ \text { H } & -2.287448 & -0.054161 & 2.599584 \\ \mathrm{C} & -2.917516 & 2.639560 & -0.038702\end{array}$




\begin{tabular}{|c|c|c|c|}
\hline $\mathrm{H}$ & -1.057907 & 3.312447 & 0.521335 \\
\hline C & -3.866609 & 1.672883 & 0.099672 \\
\hline$N$ & -4.500572 & -0.437533 & 1.088299 \\
\hline $\mathrm{H}$ & -2.980996 & 3.410606 & -0.795220 \\
\hline $\mathrm{H}$ & -4.695711 & 1.666245 & -0.591106 \\
\hline C & -4.137442 & -1.601504 & 1.889498 \\
\hline C & -5.549376 & -0.587284 & 0.081392 \\
\hline $\mathrm{H}$ & -4.024897 & -1.328921 & 2.943298 \\
\hline $\mathrm{H}$ & -4.937355 & -2.336803 & 1.819828 \\
\hline $\mathrm{H}$ & -3.214769 & -2.048738 & 1.501775 \\
\hline $\mathrm{H}$ & -5.107885 & -0.636196 & -0.920511 \\
\hline $\mathrm{H}$ & -6.091238 & -1.510618 & 0.278856 \\
\hline $\mathrm{H}$ & -6.262648 & 0.240963 & 0.144018 \\
\hline O & 0.447619 & 4.163651 & -0.105225 \\
\hline$S$ & 1.575532 & 3.297946 & -0.453934 \\
\hline O & 1.697196 & 2.778991 & -1.801193 \\
\hline O & 1.562655 & 2.118754 & 0.603623 \\
\hline C & 3.063605 & 4.169959 & -0.021828 \\
\hline $\mathrm{H}$ & 3.116739 & 5.051592 & -0.661508 \\
\hline $\mathrm{H}$ & 3.006948 & 4.456856 & 1.027805 \\
\hline $\mathrm{H}$ & 3.911257 & 3.509788 & -0.206777 \\
\hline $\mathrm{H}$ & 1.743367 & 1.212677 & 0.203087 \\
\hline $\mathrm{O}$ & -1.125717 & 0.094344 & -0.865108 \\
\hline $\mathrm{H}$ & -0.684698 & 1.763187 & 2.222925 \\
\hline $\mathrm{H}$ & -0.295949 & -0.446864 & 0.351889 \\
\hline $\mathrm{O}$ & 1.202429 & -2.344232 & -0.223827 \\
\hline C & 0.548480 & -3.487477 & 0.338524 \\
\hline $\mathrm{H}$ & -0.342302 & -3.156766 & 0.881886 \\
\hline $\mathrm{H}$ & 1.238180 & -3.996742 & 1.024801 \\
\hline C & 0.162399 & -4.412147 & -0.795288 \\
\hline $\mathrm{H}$ & -0.237683 & -5.343779 & -0.383981 \\
\hline $\mathrm{H}$ & -0.613684 & -3.950999 & -1.408323 \\
\hline $\mathrm{H}$ & 1.032865 & -4.653288 & -1.412420 \\
\hline$S$ & -1.980901 & -0.926233 & -1.563793 \\
\hline $\mathrm{O}$ & -3.187936 & -0.326434 & $-2.16291 \subseteq$ \\
\hline O & 3.583349 & -2.179518 & 1.289807 \\
\hline C & 2.667943 & -0.449058 & 2.714723 \\
\hline $\mathrm{H}$ & 1.746089 & -0.079475 & 3.167739 \\
\hline $\mathrm{H}$ & 3.355074 & -0.767488 & 3.501972 \\
\hline $\mathrm{H}$ & 3.129980 & 0.369092 & 2.155383 \\
\hline
\end{tabular}



C $\quad-0.962937 \quad-1.502108-2.916313$
H $\quad-0.032912 \quad-1.886002-2.494202$
H $\quad-1.505697 \quad-2.283409 \quad-3.450105$
H $\quad-0.767096-0.653794 \quad-3.572732$
$\begin{array}{llll}\text { O } & -2.247593 & -2.094185 & -0.693622\end{array}$

TS2 I2

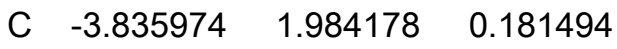
$\begin{array}{llll}\text { O } & -1.995541 & 0.402912 & -0.322027\end{array}$
$\begin{array}{llll}\text { C } & -2.118497 & 1.584152 & 1.875177\end{array}$
$\begin{array}{llll}\text { C } & -3.265570 & 0.906509 & -0.743948\end{array}$
$\begin{array}{llll}\text { O } & -4.806656 & 2.606129 & -0.172684\end{array}$
$\begin{array}{llll}\mathrm{H} & -1.688801 & 2.313981 & 2.569242\end{array}$
$\begin{array}{llll}\text { C } & -1.079249 & 1.381869 & 0.772291\end{array}$
$\begin{array}{llll}\mathrm{O} & -0.048673 & 0.730558 & 1.073313\end{array}$
$\begin{array}{llll}\text { C } & -4.272191 & -0.223647 & -0.923593\end{array}$
H $\quad-5.201548 \quad 0.192652 \quad-1.315188$
H $\quad-3.873245 \quad-0.962053 \quad-1.623252$
H $\quad-4.480423 \quad-0.715187 \quad 0.031777$
H $\quad-3.098551 \quad 1.410243 \quad-1.699284$
C $\quad \begin{array}{llll}1.313225 & -1.997835 & 1.401189\end{array}$
$\begin{array}{llll}\text { C } & 2.338575 & -1.159618 & 1.740912\end{array}$
$\begin{array}{llll}N & 1.426212 & -2.859688 & 0.374076\end{array}$
C $3.525251 \quad-1.139862 \quad 0.959624$
H $\quad 2.167877 \quad-0.445785 \quad 2.532978$
$\begin{array}{llll}\text { C } & 2.560362 & -2.921738 & -0.349437\end{array}$
H $\quad 0.582779 \quad-3.406730 \quad 0.089427$
$\begin{array}{llll}\text { C } & 3.624021 & -2.108379 & -0.078889\end{array}$
$\begin{array}{llll}\mathrm{N} & 4.490604 & -0.224017 & 1.165029\end{array}$
H $2.563708 \quad-3.628477 \quad-1.169625$
H $4.489037 \quad-2.158951 \quad-0.723568$
$\begin{array}{llll}\text { C } & 4.258642 & 0.874955 & 2.095158\end{array}$
$\begin{array}{llll}\text { C } & 5.637297 & -0.157705 & 0.265143\end{array}$
H $\quad 4.100142 \quad 0.501153 \quad 3.111910$
H $5.138579 \quad 1.515850 \quad 2.107309$
H $3.399708 \quad 1.477745 \quad 1.778625$
$\begin{array}{llll}\text { H } & 5.315376 & 0.071001 & -0.756719\end{array}$
$\begin{array}{llll}H & 6.305077 & 0.631546 & 0.606737\end{array}$ 

H $\quad 6.196402 \quad-1.098929 \quad 0.281666$
$\begin{array}{lllll}\text { O } & -0.855936 & -4.090302 & -0.329244\end{array}$
S $\quad-2.000123 \quad-3.159039 \quad-0.524837$
$\begin{array}{lllll}\mathrm{O} & -2.228762 & -2.725303 & -1.904479\end{array}$
$\begin{array}{lllll}\mathrm{O} & -1.900220 & -2.001679 & 0.440898\end{array}$
$\begin{array}{llll}\text { C } & -3.440822 & -4.071500 & 0.000938\end{array}$

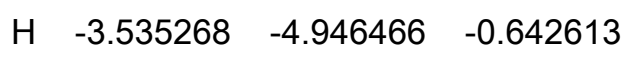

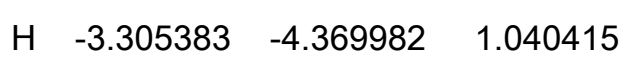
H $\quad-4.311068 \quad-3.422983 \quad-0.107239$

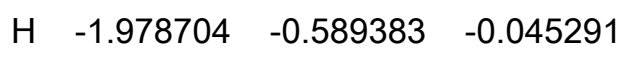
$\begin{array}{lllll}\mathrm{O} & 1.384602 & 0.142098 & -0.861342\end{array}$
$\begin{array}{llll}H & 0.346973 & -1.973271 & 1.889536\end{array}$
H $\quad 0.7964490 .463935 \quad-0.049124$
$\begin{array}{llll}\mathrm{O} & -0.967811 & 2.454158 & -0.104202\end{array}$
$\begin{array}{llll}\text { C } & -0.432461 & 3.644108 & 0.498973\end{array}$
$\begin{array}{llll}H & 0.480396 & 3.384493 & 1.045617\end{array}$
$\begin{array}{llll}H & -1.176680 & 4.058852 & 1.190685\end{array}$
$\begin{array}{llll}\text { C } & -0.130600 & 4.637357 & -0.601142\end{array}$
H $\quad 0.169951 \quad 5.590802 \quad-0.157247$
H $\quad 0.691661 \quad 4.281241 \quad-1.224627$
H $\quad-1.015746 \quad 4.809360 \quad-1.219940$
S $\quad 2.258432 \quad 1.275664 \quad-1.490083$
$\begin{array}{lllll}\text { O } & 3.428418 & 0.649669 & -2.094434\end{array}$
$\begin{array}{llll}\mathrm{O} & -3.294628 & 2.241517 & 1.381796\end{array}$
$\begin{array}{llll}\text { C } & -2.471029 & 0.326372 & 2.646922\end{array}$
H $\quad-1.564439 \quad-0.098542 \quad 3.082262$
H $\quad-3.169732 \quad 0.580270 \quad 3.447211$
$\begin{array}{llll}H & -2.921822 & -0.434287 & 2.006213\end{array}$
$\begin{array}{llll}\text { C } & 1.206017 & 1.887494 & -2.788274\end{array}$
H $\quad 0.268695 \quad 2.208708 \quad-2.331372$
H $\quad 1.718471 \quad 2.719080-3.273665$
H $\quad 1.036917 \quad 1.072668 \quad-3.492171$
$\begin{array}{llll}\text { O } & 2.472776 & 2.341131 & -0.511458\end{array}$

Complex 12
$\begin{array}{llll}\text { C } & 4.294554 & -1.661501 & 0.715407\end{array}$
$\begin{array}{llll}\text { O } & 2.657888 & -0.380535 & -0.543882\end{array}$
$\begin{array}{llll}\text { C } & 2.009648 & -1.754557 & 1.742119\end{array}$ 


\begin{tabular}{|c|c|c|c|}
\hline C & 4.025667 & -0.523580 & -0.271669 \\
\hline C & 5.403868 & -2.112927 & 0.849713 \\
\hline & 1.625422 & -2.535584 & 2.412883 \\
\hline & 0.967180 & -1.693397 & 0.623143 \\
\hline & 0.046839 & -0.893138 & 0.730748 \\
\hline & 4.722555 & 0.757514 & 0.186284 \\
\hline & 5.784122 & 0.555539 & 0.343163 \\
\hline & 4.604753 & 1.519081 & -0.588389 \\
\hline & 4.288752 & 1.136016 & 1.116007 \\
\hline r & 4.526697 & -0.864963 & -1.188311 \\
\hline C & -1.259766 & 1.887247 & 0.865699 \\
\hline C & -2.332554 & 1.268762 & 1.449665 \\
\hline N & -1.388274 & 2.561761 & -0.291329 \\
\hline & -3.605487 & 1.304741 & 0.818933 \\
\hline H & -2.163470 & 0.696937 & 2.350306 \\
\hline C & -2.576912 & 2.630999 & -0.917598 \\
\hline $\mathrm{H}$ & -0.542296 & 3.035275 & -0.699262 \\
\hline C & -3.691552 & 2.029189 & -0.403297 \\
\hline$N$ & -4.674207 & 0.670872 & 1.338390 \\
\hline $\mathrm{H}$ & -2.590716 & 3.177426 & -1.852360 \\
\hline $\mathrm{H}$ & -4.612464 & 2.080084 & -0.965459 \\
\hline C & -4.532376 & -0.121335 & 2.552650 \\
\hline C & -5.916915 & 0.595990 & 0.580042 \\
\hline $\mathrm{H}$ & -4.153904 & 0.494856 & 3.374885 \\
\hline $\mathrm{H}$ & -5.511928 & -0.498476 & 2.842770 \\
\hline $\mathrm{H}$ & -3.864964 & -0.972977 & 2.383138 \\
\hline $\mathrm{H}$ & -5.749537 & 0.110208 & -0.387149 \\
\hline $\mathrm{H}$ & -6.636726 & 0.004236 & 1.143234 \\
\hline $\mathrm{H}$ & -6.344715 & 1.593301 & 0.428707 \\
\hline O & 0.762535 & 3.850152 & -1.209233 \\
\hline$S$ & 2.052338 & 3.229430 & -0.782281 \\
\hline O & 2.966800 & 2.891603 & -1.874761 \\
\hline $\mathrm{O}$ & 1.790713 & 2.082709 & 0.151316 \\
\hline C & 2.864697 & 4.471306 & 0.214564 \\
\hline $\mathrm{H}$ & 3.048673 & 5.341814 & -0.415618 \\
\hline $\mathrm{H}$ & 2.215320 & 4.732228 & 1.050567 \\
\hline $\mathrm{H}$ & 3.806839 & 4.054023 & 0.572519 \\
\hline $\mathrm{H}$ & 2.360459 & 0.546580 & -0.396768 \\
\hline $\mathrm{O}$ & -1.742162 & -0.655502 & -1.119216 \\
\hline $\mathrm{H}$ & -0.250777 & 1.838039 & 1.258879 \\
\hline
\end{tabular}




$\begin{array}{lrrr}\mathrm{H} & -1.034026 & -0.785543 & -0.405213 \\ \mathrm{O} & 0.867921 & -2.615449 & -0.321945 \\ \mathrm{C} & 1.921710 & -3.521193 & -0.714443 \\ \mathrm{H} & 2.305657 & -4.048548 & 0.160112 \\ \mathrm{H} & 2.722804 & -2.928596 & -1.160748 \\ \mathrm{C} & 1.322828 & -4.474168 & -1.724593 \\ \mathrm{H} & 2.096693 & -5.163288 & -2.073549 \\ \mathrm{H} & 0.516430 & -5.063898 & -1.279078 \\ \mathrm{H} & 0.932984 & -3.929224 & -2.588409 \\ \mathrm{~S} & -2.918228 & -1.696146 & -0.956563 \\ \mathrm{O} & -4.079956 & -1.132586 & -1.626039 \\ \mathrm{O} & 3.289531 & -2.277910 & 1.394701 \\ \mathrm{C} & 2.086954 & -0.452895 & 2.525932 \\ \mathrm{H} & 1.140788 & -0.278186 & 3.041149 \\ \mathrm{H} & 2.880890 & -0.541609 & 3.271538 \\ \mathrm{H} & 2.276381 & 0.403003 & 1.876331 \\ \mathrm{C} & -2.330665 & -3.089159 & -1.894136 \\ \mathrm{H} & -1.388740 & -3.413411 & -1.450682 \\ \mathrm{H} & -3.086911 & -3.872205 & -1.829887 \\ \mathrm{H} & -2.187480 & -2.769847 & -2.926268 \\ \mathrm{O} & -3.031578 & -2.098549 & 0.441201\end{array}$


For DMAP:MSA (1:1) - Propagation

\begin{tabular}{|c|c|c|c|}
\hline & -1.590688 & 2.809564 & -0. \\
\hline & 0.377192 & -2.396415 & -1.74803 \\
\hline & 0.484553 & -2.341103 & 0.66024 \\
\hline & -0.973664 & -1.941418 & -1.49524 \\
\hline & -2.702985 & -3.273659 & -0.43938 \\
\hline & 0.277139 & -1.272592 & 0.80020 \\
\hline & 1.177667 & -2.532556 & -0.6 \\
\hline & 2.34 & & \\
\hline & -1.741208 & -1.974140 & -2.79306 \\
\hline & -2.745266 & -1.580819 & -2.62177 \\
\hline & -1.234452 & & \\
\hline & -1.824534 & -2.5 & \\
\hline & -0.912484 & -0.918801 & 728 \\
\hline & 4.243654 & 2.279404 & 5098 \\
\hline & 5.206795 & 1.397298 & -0.59 \\
\hline & 3.236281 & & \\
\hline & 5.112522 & & \\
\hline & 5.9 & & \\
\hline & 3.15 & & \\
\hline & 2.404846 & 2.50 & \\
\hline & 4.077208 & -0.3 & $-1 . \varepsilon$ \\
\hline & 5.940002 & -0.900860 & \\
\hline & 2.298878 & & \\
\hline & 3.925312 & -1.33 & -2 \\
\hline & 6.909110 & -0.5 & \\
\hline & 5.738270 & -2.3 & -0.785583 \\
\hline & 6.401528 & & \\
\hline & 7.451655 & & \\
\hline & 7.638190 & & \\
\hline & 4.728737 & -2.636266 & \\
\hline & 5.900299 & -2.493108 & -1 \\
\hline & 6.460786 & -2.904743 & -0.228998 \\
\hline & 0.785820 & 3.100328 & -1.718388 \\
\hline & -0.135855 & 2.123726 & -1.085623 \\
\hline & -0.831949 & 2.612365 & $0.11 s$ \\
\hline & 0.554220 & 0.809250 & -0.83268 \\
\hline
\end{tabular}




\begin{tabular}{|c|c|c|c|}
\hline C & -1.378762 & 1.762896 & -2.310254 \\
\hline $\mathrm{H}$ & -1.864339 & 2.704293 & -2.569627 \\
\hline $\mathrm{H}$ & -0.885185 & 1.337394 & -3.184639 \\
\hline $\mathrm{H}$ & -2.094364 & 1.066704 & -1.872580 \\
\hline $\mathrm{H}$ & -0.984574 & 1.215107 & 1.269809 \\
\hline C & 2.362183 & 0.117689 & 0.854862 \\
\hline $\mathrm{H}$ & 4.213838 & 3.303140 & -0.646733 \\
\hline $\mathrm{H}$ & 1.649002 & 0.553634 & 0.283889 \\
\hline O & -1.032002 & 0.466106 & 1.900842 \\
\hline 0 & -2.370350 & 0.254508 & 2.257132 \\
\hline $\mathrm{H}$ & -2.380607 & -0.532359 & 3.017743 \\
\hline$S$ & 2.906600 & 1.011446 & 2.047222 \\
\hline 0 & 2.772714 & 2.418432 & 1.699491 \\
\hline O & -0.782617 & -3.037687 & 0.638105 \\
\hline & 1.287673 & -2.877830 & 1.820084 \\
\hline$r$ & 2.249429 & -2.363591 & 1.854033 \\
\hline $\mathrm{H}$ & 0.743369 & -2.697976 & 2.749938 \\
\hline $\mathrm{H}$ & 1.463871 & -3.950054 & 1.708743 \\
\hline C & 1.796205 & 0.626764 & 3.379738 \\
\hline $\mathrm{H}$ & 1.929371 & -0.426105 & 3.629286 \\
\hline $\mathrm{H}$ & 2.079606 & 1.260706 & 4.220932 \\
\hline $\mathrm{H}$ & 0.771420 & 0.820332 & 3.053936 \\
\hline 0 & 4.221719 & 0.475906 & 2.373642 \\
\hline C & -5.169839 & -1.236201 & 0.351860 \\
\hline $\mathrm{H}$ & -4.618812 & -1.876102 & -0.341448 \\
\hline C & -3.037373 & 1.520020 & 2.805559 \\
\hline $\mathrm{H}$ & -3.016734 & 2.310081 & 2.048722 \\
\hline $\mathrm{H}$ & -4.075444 & 1.325565 & 3.085953 \\
\hline $\mathrm{H}$ & -2.483925 & 1.864730 & 3.682094 \\
\hline C & -6.338071 & -1.985838 & 0.968653 \\
\hline $\mathrm{H}$ & -5.968393 & -2.876812 & 1.480181 \\
\hline $\mathrm{H}$ & -6.870417 & -1.355552 & 1.685983 \\
\hline $\mathrm{H}$ & -7.027920 & -2.293670 & 0.179656 \\
\hline C & -5.872322 & 2.340389 & -0.402029 \\
\hline $\mathrm{H}$ & -6.741036 & 2.194884 & -1.048588 \\
\hline $\mathrm{H}$ & -6.127163 & 3.034264 & 0.400531 \\
\hline C & -4.662419 & 2.812278 & -1.186613 \\
\hline $\mathrm{H}$ & -4.449684 & 2.123990 & -2.007953 \\
\hline H & -4.862435 & 3.801995 & -1.608448 \\
\hline 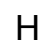 & -3.779639 & 2.878610 & -0.544063 \\
\hline
\end{tabular}



C $\quad-3.167567 \quad-0.245743 \quad 1.061565$
C $\quad-5.640159 \quad-0.018586-0.436310$
$\begin{array}{llll}\mathrm{O} & -4.295257 & -0.855811 & 1.425375\end{array}$
$\begin{array}{llll}\text { O } & -5.585159 & 1.105104 & 0.281505\end{array}$
$\begin{array}{llll}\text { O } & -6.052680 & -0.099616 & -1.570355\end{array}$
$\begin{array}{llll}\text { O } & -2.844755 & -0.072945 & -0.095030\end{array}$

TSP1
C $\quad-0.053885 \quad-4.249565 \quad-0.664764$
$\begin{array}{llll}\mathrm{O} & -1.226914 & -2.195076 & 0.057653\end{array}$
$\begin{array}{llll}\text { C } & -0.763396 & -2.543198 & -2.245890\end{array}$
C $\quad-0.597928 \quad-3.403312 \quad 0.487430$
O $\quad 0.459725 \quad-5.318182 \quad-0.448991$
H $\quad-0.216780 \quad-2.160605 \quad-3.110971$
$\begin{array}{llll}\text { C } & -0.605929 & -1.571346 & -1.069821\end{array}$
$\begin{array}{lllll}\mathrm{O} & -1.263084 & -0.425573 & -1.339897\end{array}$
$\begin{array}{llll}\text { C } & -1.601319 & -4.192688 & 1.312218\end{array}$
H $\quad-1.128652 \quad-5.101998 \quad 1.685669$
$\begin{array}{llll}\mathrm{H} & -1.936863 & -3.584081 & 2.155176\end{array}$
$\begin{array}{llll}\text { H } & -2.464290 & -4.475915 & 0.699367\end{array}$
H $\quad 0.272977 \quad-3.138759 \quad 1.096053$
C $\quad-3.682870 \quad 1.547232 \quad-1.017382$
$\begin{array}{llll}\text { C } & -2.931729 & 2.461380 & -1.696894\end{array}$
$\begin{array}{llll}N & -4.136065 & 1.799827 & 0.226598\end{array}$
$\begin{array}{llll}\text { C } & -2.565352 & 3.687715 & -1.071757\end{array}$
$\begin{array}{llll}H & -2.542023 & 2.181088 & -2.664067\end{array}$
$\begin{array}{llll}\text { C } & -3.839161 & 2.962193 & 0.846354\end{array}$
$\begin{array}{llll}\mathrm{H} & -4.625691 & 1.065259 & 0.745034\end{array}$
$\begin{array}{llll}C & -3.091097 & 3.920214 & 0.232876\end{array}$
$\begin{array}{llll}\mathrm{N} & -1.746087 & 4.569147 & -1.663166\end{array}$
H $\quad-4.202023 \quad 3.069227 \quad 1.860107$
$\mathrm{H} \quad-2.829038 \quad 4.803655 \quad 0.794451$
$\begin{array}{llll}\text { C } & -1.097979 & 4.220619 & -2.921506\end{array}$
C $\quad-1.202123 \quad 5.682015 \quad-0.887548$
H $\quad-1.843707 \quad 3.987942 \quad-3.687810$
$\begin{array}{llll}H & -0.523024 & 5.078008 & -3.268173\end{array}$
H $\quad-0.418962 \quad 3.373931 \quad-2.770465$
H $\quad-0.678170 \quad 5.305225 \quad-0.001788$ 


\begin{tabular}{|c|c|c|c|}
\hline & -0.496399 & 6.228075 & \\
\hline & -1.998678 & 6.373655 & -0.593351 \\
\hline & -5.272965 & -0.342798 & \\
\hline & -4.392754 & 1.512228 & 1.73217 \\
\hline & -3.380175 & -1.631397 & 2.76162 \\
\hline & -3.739719 & -1.589760 & 0.29027 \\
\hline & -5.428786 & -2.956508 & 49613 \\
\hline & -5.980973 & -2.943991 & \\
\hline & -6.110037 & -2.906765 & 0.9 \\
\hline & -4.787597 & -3.8 & 605 \\
\hline & -2.750899 & -1.789630 & 0.3 \\
\hline & -1.030810 & 1.23 & \\
\hline & -3.907284 & 0.566637 & -1.416142 \\
\hline & -1.109236 & & \\
\hline & 0.717412 & -1.398694 & \\
\hline & 1.759414 & -1.251091 & -1.6 \\
\hline & 1.803844 & -2.135009 & -2.25672 \\
\hline & 0.058739 & 2.270951 & 0.609588 \\
\hline & -0.294487 & 3.463479 & 1.40 \\
\hline & & & \\
\hline & -2.209983 & $-2.7 \varepsilon$ & \\
\hline & -2.672811 & -1.8 & \\
\hline & -2.247864 & -3.508437 & -3 \\
\hline & -2.778756 & -3.189500 & -1.788982 \\
\hline & 1.446641 & 1.498557 & 1.429626 \\
\hline & & & \\
\hline & 2.309180 & & \\
\hline & 1.181948 & 1.362766 & 2.4 \\
\hline & 0.499443 & 2.574446 & -0.771322 \\
\hline & 1.664218 & 0.024716 & -2.43656 \\
\hline & 1.596597 & 0.907234 & \\
\hline & & 0.103957 & \\
\hline & 0.765461 & -0.000712 & -3.05 \\
\hline & 5.361672 & -1.073777 & $-0 . \varepsilon$ \\
\hline & 5.496485 & -1.974780 & \\
\hline & 6.457468 & -0.933421 & \\
\hline & 6.446289 & -1.797687 & -2.558681 \\
\hline & 6.306361 & -0.025690 & -2.480416 \\
\hline & 7.431867 & -0.882690 & -1.398854 \\
\hline & 6.098825 & 0.949776 & 2.15996 \\
\hline
\end{tabular}




$\begin{array}{rrrr}\mathrm{H} & 6.066599 & 1.937337 & 1.695493 \\ \mathrm{H} & 7.030470 & 0.827383 & 2.713134 \\ \mathrm{C} & 4.894982 & 0.744371 & 3.071027 \\ \mathrm{H} & 4.930396 & -0.260685 & 3.507572 \\ \mathrm{H} & 3.976558 & 0.825968 & 2.481246 \\ \mathrm{O} & 4.118347 & -1.153013 & -1.547497 \\ \mathrm{O} & 6.114632 & -0.071330 & 1.147203 \\ \mathrm{O} & 4.958338 & 1.722708 & 4.095013 \\ \mathrm{H} & 4.106454 & 2.154824 & 4.179884 \\ \mathrm{C} & 3.032710 & -1.291679 & -0.769346 \\ \mathrm{C} & 5.327883 & 0.133625 & 0.087270 \\ \mathrm{O} & 3.086658 & -1.434388 & 0.427700 \\ \mathrm{O} & 4.700131 & 1.141992 & -0.127909\end{array}$

\section{Int. $P 1$}

$\begin{array}{llll}\text { C } & -0.591438 & -3.804783 & 0.298468\end{array}$

$\begin{array}{llll}\mathrm{O} & -1.335885 & -1.641013 & 1.289603\end{array}$

$\begin{array}{llll}\text { C } & -1.267384 & -1.930283 & -1.069572\end{array}$

$\begin{array}{llll}\text { C } & -0.892926 & -2.966678 & 1.543544\end{array}$

$\begin{array}{llll}\mathrm{O} & -0.198003 & -4.940366 & 0.412209\end{array}$

H $\quad-0.855729 \quad-1.551621 \quad-2.006371$

$\begin{array}{llll}\text { C } & -0.824880 & -1.070610 & 0.122800\end{array}$

$\begin{array}{llll}\mathrm{O} & -1.292098 & 0.192805 & 0.112810\end{array}$

$\begin{array}{llll}\text { C } & -1.948345 & -3.642132 & 2.406387\end{array}$

H $\quad-1.618108 \quad-4.644539 \quad 2.683063$

$\begin{array}{llll}\mathrm{H} & -2.105199 & -3.050562 & 3.311762\end{array}$

$\begin{array}{llll}H & -2.896507 & -3.719396 & 1.865604\end{array}$

H $\quad 0.058349 \quad-2.926598 \quad 2.088448$

$\begin{array}{llll}\text { C } & -2.549132 & 1.325896 & 2.730276\end{array}$

$\begin{array}{llll}\text { C } & -3.582539 & 0.535123 & 2.321472\end{array}$

$\begin{array}{llll}\mathrm{N} & -2.143668 & 2.378305 & 1.993017\end{array}$

$\begin{array}{llll}\text { C } & -4.209776 & 0.781145 & 1.067758\end{array}$

H $\quad-3.851934 \quad-0.310885 \quad 2.936296$

$\begin{array}{llll}\text { C } & -2.732060 & 2.667304 & 0.811876\end{array}$

H $\quad-1.296617 \quad 2.861339 \quad 2.297264$

$\begin{array}{llll}\text { C } & -3.773715 & 1.922932 & 0.340005\end{array}$

$\begin{array}{llll}\mathrm{N} & -5.153167 & -0.044876 & 0.581805\end{array}$

H $\quad-2.304265 \quad 3.481670 \quad 0.242639$ 

H $\quad-4.151458 \quad 2.143701 \quad-0.649879$
$\begin{array}{llll}\text { C } & -5.540052 & -1.227385 & 1.333329\end{array}$
$\begin{array}{llll}\text { C } & -5.819377 & 0.251405 & -0.686137\end{array}$
$\begin{array}{llll}\text { H } & -6.037398 & -0.965562 & 2.275429\end{array}$
$\begin{array}{llll}H & -6.231624 & -1.817895 & 0.734701\end{array}$
H $\quad-4.666018-1.849867 \quad 1.554329$
$\begin{array}{llll}\text { H } & -6.450586 & 1.143241 & -0.594238\end{array}$
H $\quad-5.094474 \quad 0.410060 \quad-1.490485$
H $\quad-6.453401 \quad-0.592918-0.952796$
$\begin{array}{llll}\mathrm{O} & 0.439273 & 2.747173 & 3.163286\end{array}$
S $\quad 1.305535 \quad 1.906115 \quad 2.331485$
$\begin{array}{llll}\text { O } & 1.439634 & 2.253738 & 0.926813\end{array}$
$\begin{array}{llll}0 & 0.762796 & 0.433750 & 2.500930\end{array}$
$\begin{array}{llll}\text { C } & 2.911220 & 1.824808 & 3.081270\end{array}$
H $\quad 3.352221 \quad 2.820057 \quad 3.015070$
$\mathrm{H} \quad 2.788286 \quad 1.520461 \quad 4.120357$
H $3.492685 \quad 1.0934092 .518426$
H $\quad 0.864131 \quad-0.105356 \quad 1.663429$
$\begin{array}{llll}\text { O } & -1.441141 & 1.754154 & -1.958053\end{array}$
H $\quad-1.987880 \quad 1.140315 \quad 3.636617$
$\begin{array}{llll}\mathrm{H} & -1.254811 & 0.676272 & -0.760521\end{array}$
$\begin{array}{llll}\text { O } & 0.606212 & -1.098864 & 0.321045\end{array}$
C $\quad \begin{array}{llll}1.512958 & -1.088381 & -0.778741\end{array}$
H $\quad 1.386641 \quad-2.007320 \quad-1.361277$
S $\quad-2.238149 \quad 1.251239 \quad-3.127155$
$\begin{array}{lllll}\text { O } & -3.694949 & 1.301982 & -2.866589\end{array}$
$\begin{array}{lllll}\mathrm{O} & -0.719902 & -3.258522 & -0.917254\end{array}$
C $\quad-2.777575 \quad-1.999130 \quad-1.200669$
H $\quad-3.180097 \quad-1.005313 \quad-1.401186$
H $\quad-3.030660 \quad-2.641741 \quad-2.045632$
H $\quad-3.230929 \quad-2.395925 \quad-0.287779$
$\begin{array}{llll}\text { C } & -1.922889 & 2.428042 & -4.435038\end{array}$
H $\quad-0.852107 \quad 2.433270 \quad-4.639971$
H $\quad-2.481171 \quad 2.107533 \quad-5.315080$
H $\quad-2.257937 \quad 3.411509 \quad-4.104678$
$\begin{array}{llll}\mathrm{O} & -1.742306 & -0.056643 & -3.598147\end{array}$
C $\quad 5.157107 \quad-1.576243 \quad-0.563959$
H $\quad \begin{array}{llll}5.255919 & -1.965873 & 0.452259\end{array}$
$\begin{array}{llll}\text { C } & 1.418988 & 0.144777 & -1.678219\end{array}$
H $1.451809 \quad 1.052877-1.073630$ 


$\begin{array}{lrrr}\mathrm{H} & 2.262176 & 0.138471 & -2.373888 \\ \mathrm{H} & 0.497420 & 0.144261 & -2.262365 \\ \mathrm{C} & 5.963330 & -2.407602 & -1.549135 \\ \mathrm{H} & 5.618183 & -3.443313 & -1.526193 \\ \mathrm{H} & 5.847521 & -2.019885 & -2.564448 \\ \mathrm{H} & 7.019117 & -2.377698 & -1.271708 \\ \mathrm{C} & 5.265287 & 2.086125 & -1.248896 \\ \mathrm{H} & 6.350790 & 2.166041 & -1.344351 \\ \mathrm{H} & 4.788495 & 2.477968 & -2.148316 \\ \mathrm{C} & 4.755550 & 2.781132 & -0.001623 \\ \mathrm{H} & 5.264285 & 2.395111 & 0.885558 \\ \mathrm{H} & 4.960684 & 3.853671 & -0.074936 \\ \mathrm{H} & 3.676869 & 2.639553 & 0.105979 \\ \mathrm{C} & 2.888566 & -1.119112 & -0.139297 \\ \mathrm{C} & 5.660299 & -0.135943 & -0.527871 \\ \mathrm{O} & 3.786827 & -1.653679 & -0.970065 \\ \mathrm{O} & 4.903018 & 0.688969 & -1.245592 \\ \mathrm{O} & 6.671200 & 0.173057 & 0.060555 \\ \mathrm{O} & 3.158535 & -0.678121 & 0.956110\end{array}$

\section{Int. P2}

C $\quad 0.212079-4.177425 \quad-0.698633$

$\begin{array}{llll}\mathrm{O} & -1.071800 & -2.190497 & 0.021903\end{array}$

C $\quad-0.461313 \quad-2.438210 \quad-2.259864$

$\begin{array}{llll}\text { C } & -0.425489 & -3.390557 & 0.447731\end{array}$

$\begin{array}{llll}\text { O } & 0.747209 & -5.237013 & -0.489830\end{array}$

H $\quad 0.121868 \quad-2.007084 \quad-3.076924$

C $\quad-0.407000 \quad-1.505134 \quad-1.043658$

$\begin{array}{lllll}\mathrm{O} & -1.086304 & -0.372537 & -1.312558\end{array}$

$\begin{array}{llll}\text { C } & -1.445137 & -4.240976 & 1.187474\end{array}$

H $\quad-0.962493 \quad-5.146232 \quad 1.558015$

H $\quad-1.849078 \quad-3.673126 \quad 2.028891$

$\begin{array}{llll}\mathrm{H} & -2.261617 & -4.531766 & 0.517136\end{array}$

H $\quad 0.400011 \quad-3.117965 \quad 1.113461$

C $\quad-3.591856 \quad 1.499182 \quad-1.067731$

C $\quad-2.824024 \quad 2.451918-1.671370$

$\begin{array}{llll}\text { N } & -4.125719 & 1.700730 & 0.153335\end{array}$

$\begin{array}{llll}\text { C } & -2.526199 & 3.665108 & -0.986939\end{array}$ 

$\begin{array}{llll}\mathrm{H} & -2.369334 & 2.211039 & -2.620577\end{array}$
$\begin{array}{llll}\text { C } & -3.897093 & 2.849566 & 0.825107\end{array}$
H $\quad-4.622955 \quad 0.936734 \quad 0.619145$
$\begin{array}{llll}\text { C } & -3.138230 & 3.844244 & 0.288090\end{array}$
$\begin{array}{llll}\mathrm{N} & -1.691204 & 4.582041 & -1.497027\end{array}$
H $\quad-4.322263 \quad 2.915302 \quad 1.817921$
$\begin{array}{llll}\mathrm{H} & -2.933007 & 4.714550 & 0.892050\end{array}$
$\begin{array}{llll}\text { C } & -0.951463 & 4.284474 & -2.717317\end{array}$
C $\quad \begin{array}{llll}-1.222578 & 5.679548 & -0.653078\end{array}$
H $\quad-1.637707 \quad 4.058253 \quad-3.539105$
$\begin{array}{llll}H & -0.374637 & 5.163827 & -2.999968\end{array}$
H $\quad-0.264264 \quad 3.448850 \quad-2.543115$
$\begin{array}{llll}\mathrm{H} & -0.750602 & 5.284723 & 0.253835\end{array}$
$\begin{array}{llll}\mathrm{H} & -0.488472 & 6.257882 & -1.211330\end{array}$
H $\quad-2.051075 \quad 6.346541 \quad-0.391960$
$\begin{array}{llll}\mathrm{O} & -5.271268 & -0.530466 & 1.497660\end{array}$
S $\quad-4.352062 \quad-1.669254 \quad 1.530335$
$\begin{array}{llll}\text { O } & -3.400203 & -1.793048 & 2.615442\end{array}$
$\begin{array}{llll}\text { O } & -3.611517 & -1.671894 & 0.129538\end{array}$
$\begin{array}{llll}\text { C } & -5.335119 & -3.147257 & 1.433609\end{array}$
$\begin{array}{llll}H & -5.942924 & -3.188113 & 2.338158\end{array}$
H $\quad-5.965471 \quad-3.089084 \quad 0.546752$
H $\quad-4.660613 \quad-4.002355 \quad 1.385298$
$\begin{array}{llll}\mathrm{H} & -2.618473 & -1.839463 & 0.192148\end{array}$
$\begin{array}{llll}\mathrm{O} & -1.034899 & 1.205139 & 0.720874\end{array}$
H $\quad-3.7667150 .526880 \quad-1.509243$
H $\quad-1.002043 \quad 0.272589-0.532562$
$\begin{array}{lllll}\text { O } & 0.882798 & -1.306293 & -0.544543\end{array}$
C $\quad 1.971477-1.085943-1.423555$
H $\quad 2.081857 \quad-1.939155 \quad-2.108856$
$\begin{array}{llll}S & 0.021233 & 2.271433 & 0.833648\end{array}$
$\begin{array}{llll}\text { O } & -0.431066 & 3.418758 & 1.644068\end{array}$
$\begin{array}{llll}\text { O } & 0.206947 & -3.673656 & -1.946236\end{array}$
C $\quad-1.873222 \quad-2.717333 \quad-2.741103$
H $\quad-2.348337 \quad-1.785553 \quad-3.053601$
H $\quad-1.838076 \quad-3.406687 \quad-3.587674$
H $\quad-2.476477 \quad-3.165081 \quad-1.947009$
$\begin{array}{llll}\text { C } & 1.366897 & 1.503601 & 1.722683\end{array}$
H $1.630552 \quad 0.569522 \quad 1.225623$
H $2.211000 \quad 2.194385 \quad 1.716689$ 


$\begin{array}{lrrr}\mathrm{H} & 1.028959 & 1.306776 & 2.740767 \\ \mathrm{O} & 0.549791 & 2.648166 & -0.498249 \\ \mathrm{C} & 1.881286 & 0.220725 & -2.207770 \\ \mathrm{H} & 1.743001 & 1.071204 & -1.535166 \\ \mathrm{H} & 2.792832 & 0.357139 & -2.793102 \\ \mathrm{H} & 1.023749 & 0.191807 & -2.884357 \\ \mathrm{C} & 5.524781 & -0.866804 & -0.481748 \\ \mathrm{H} & 5.625760 & -1.764637 & 0.133557 \\ \mathrm{C} & 6.671770 & -0.742022 & -1.471504 \\ \mathrm{H} & 6.686016 & -1.611234 & -2.132746 \\ \mathrm{H} & 6.556457 & 0.161568 & -2.075470 \\ \mathrm{H} & 7.621223 & -0.694235 & -0.932949 \\ \mathrm{C} & 6.278057 & 1.219641 & 2.483712 \\ \mathrm{H} & 6.232591 & 2.187601 & 1.979258 \\ \mathrm{H} & 7.242508 & 1.112489 & 2.982629 \\ \mathrm{O} & 4.318755 & -0.943248 & -1.240505 \\ \mathrm{O} & 6.275321 & 0.175276 & 1.487616 \\ \mathrm{C} & 3.197980 & -1.124682 & -0.521818 \\ \mathrm{C} & 5.459888 & 0.346203 & 0.444968 \\ \mathrm{O} & 3.192932 & -1.305175 & 0.670617 \\ \mathrm{O} & 4.795576 & 1.331809 & 0.233908 \\ \mathrm{C} & 5.121486 & 1.040674 & 3.449011 \\ \mathrm{H} & 4.165970 & 1.121266 & 2.925918 \\ \mathrm{H} & 5.172292 & 0.064326 & 3.937566 \\ \mathrm{H} & 5.161647 & 1.818432 & 4.217609\end{array}$

TS P2
$\begin{array}{llll}\text { C } & 0.156738 & -4.031379 & -0.739707\end{array}$
$\begin{array}{llll}\mathrm{O} & -1.078932 & -2.077003 & 0.142512\end{array}$
$\begin{array}{llll}\text { C } & -0.569378 & -2.239443 & -2.240654\end{array}$
$\begin{array}{llll}\text { C } & -0.480411 & -3.341618 & 0.466519\end{array}$
O $\quad 0.745837 \quad-5.072155 \quad-0.586625$
H $\quad-0.006637 \quad-1.827859-3.083983$
C $\quad-0.418770-1.260472-1.070940$
$\begin{array}{llll}\mathrm{O} & -1.044344 & -0.158584 & -1.195277\end{array}$
$\begin{array}{llll}\text { C } & -1.498700 & -4.255096 & 1.132597\end{array}$
H $\quad-0.994150 \quad-5.173797 \quad 1.434545$
H $\quad-1.922918-3.759593 \quad 2.008434$ 


$$
\begin{aligned}
& \text { H } \quad-2.306726 \quad-4.509465 \quad 0.439862 \\
& \text { H } \quad 0.343689-3.123704 \quad 1.150306 \\
& \begin{array}{llll}
\text { C } & -3.599067 & 1.395564 & -0.902171
\end{array} \\
& \text { C } \quad-2.922869 \quad 2.412479-1.517005 \\
& \begin{array}{llll}
\mathrm{N} & -4.096580 & 1.537675 & 0.339678
\end{array} \\
& \begin{array}{llll}
\text { C } & -2.691895 & 3.632149 & -0.824297
\end{array} \\
& \begin{array}{llll}
\mathrm{H} & -2.488973 & 2.220458 & -2.486941
\end{array} \\
& \begin{array}{llll}
\text { C } & -3.924452 & 2.685785 & 1.023191
\end{array} \\
& \text { H } \quad-4.552886 \quad 0.7205290 .792786 \\
& \begin{array}{llll}
\text { C } & -3.253154 & 3.742762 & 0.480045
\end{array} \\
& \text { N } \quad-1.956653 \quad 4.623442 \quad-1.359566 \\
& \begin{array}{llll}
\mathrm{H} & -4.326542 & 2.706725 & 2.028001
\end{array} \\
& \text { H } \quad-3.097942 \quad 4.621160 \quad 1.088353 \\
& \text { C } \quad-1.285279 \quad 4.418232 \quad-2.636560 \\
& \text { C } \quad-1.578505 \quad 5.770809-0.541249 \\
& \text { H } \quad-2.010460 \quad 4.178213 \quad-3.420661 \\
& \text { H } \quad-0.781606 \quad 5.341025 \quad-2.920457 \\
& \text { H } \quad-0.537690 \quad 3.621856 \quad-2.552323 \\
& \text { H } \quad-1.022210 \quad 5.4455540 .344686 \\
& \mathrm{H} \quad-0.941479 \quad 6.426838 \quad-1.132130 \\
& \text { H } \quad-2.463192 \quad 6.342061 \quad-0.239755 \\
& \begin{array}{llll}
\mathrm{O} & -5.210762 & -0.675061 & 1.437372
\end{array} \\
& \text { S } \quad-4.341982-1.877353 \quad 1.431535 \\
& \begin{array}{llll}
\mathrm{O} & -3.528235 & -2.084093 & 2.627786
\end{array} \\
& \begin{array}{lllll}
\mathrm{O} & -3.517473 & -1.907562 & 0.154548
\end{array} \\
& \begin{array}{llll}
\text { C } & -5.448989 & -3.266001 & 1.270967
\end{array} \\
& \begin{array}{llll}
\text { H } & -6.104002 & -3.269665 & 2.142538
\end{array} \\
& \text { H } \quad-6.026747 \quad-3.148207 \quad 0.354420 \\
& \text { H } \quad-4.849675 \quad-4.176553 \quad 1.240332 \\
& \text { H } \quad-2.154335 \quad-2.006180 \quad 0.183406 \\
& \begin{array}{llll}
\text { O } & -0.891309 & 1.360879 & 0.721932
\end{array} \\
& \begin{array}{llll}
\mathrm{H} & -3.721637 & 0.417317 & -1.349121
\end{array} \\
& \text { H } \quad-0.889898 \quad 0.704895 \quad-0.138926 \\
& \begin{array}{lllll}
\text { O } & 0.880779 & -1.210979 & -0.542325
\end{array} \\
& \text { C } 1.981331-1.092343 \quad-1.427264 \\
& \text { H } \quad 2.024329-1.962818-2.098066 \\
& \text { S } \quad 0.236174 \quad 2.436109 \quad 0.726182 \\
& \begin{array}{llll}
\text { O } & -0.197352 & 3.544165 & 1.573451
\end{array} \\
& \begin{array}{lllll}
\text { O } & 0.083538 & -3.493004 & -1.967538
\end{array} \\
& \begin{array}{llll}
\text { C } & -2.004799 & -2.469178 & -2.673278
\end{array}
\end{aligned}
$$




$\begin{array}{lrrr}\mathrm{H} & -2.457012 & -1.514662 & -2.949037 \\ \mathrm{H} & -2.017713 & -3.137947 & -3.536840 \\ \mathrm{H} & -2.603884 & -2.909879 & -1.873286 \\ \mathrm{C} & 1.593520 & 1.606842 & 1.523436 \\ \mathrm{H} & 1.745059 & 0.640423 & 1.041741 \\ \mathrm{H} & 2.479611 & 2.233614 & 1.415028 \\ \mathrm{H} & 1.329470 & 1.468541 & 2.572067 \\ \mathrm{O} & 0.629957 & 2.756696 & -0.648275 \\ \mathrm{C} & 1.985218 & 0.202235 & -2.237959 \\ \mathrm{H} & 1.989393 & 1.076736 & -1.584880 \\ \mathrm{H} & 2.866988 & 0.225693 & -2.881040 \\ \mathrm{H} & 1.087718 & 0.260532 & -2.857839 \\ \mathrm{C} & 5.537569 & -1.005307 & -0.482976 \\ \mathrm{H} & 5.608594 & -1.885780 & 0.161118 \\ \mathrm{C} & 6.692596 & -0.949325 & -1.470149 \\ \mathrm{H} & 6.679793 & -1.839378 & -2.102885 \\ \mathrm{H} & 6.610398 & -0.062948 & -2.104334 \\ \mathrm{H} & 7.640662 & -0.916107 & -0.928553 \\ \mathrm{C} & 6.425115 & 1.178332 & 2.373676 \\ \mathrm{H} & 6.357896 & 2.129472 & 1.840553 \\ \mathrm{H} & 7.412819 & 1.083530 & 2.827151 \\ \mathrm{O} & 4.333071 & -1.066020 & -1.243806 \\ \mathrm{O} & 6.371057 & 0.101993 & 1.413559 \\ \mathrm{C} & 3.204458 & -1.191678 & -0.526417 \\ \mathrm{C} & 5.506094 & 0.236111 & 0.407690 \\ \mathrm{O} & 3.188121 & -1.357086 & 0.668391 \\ \mathrm{O} & 4.821753 & 1.210620 & 0.202842 \\ \mathrm{C} & 5.318871 & 1.035866 & 3.401873 \\ \mathrm{H} & 4.338006 & 1.106248 & 2.926280 \\ \mathrm{H} & 5.390068 & 0.074779 & 3.917213 \\ \mathrm{H} & 5.402255 & 1.836039 & 4.143400\end{array}$

Complex P2
C $\quad 2.128072 \quad-2.413326 \quad-0.892477$
O $\quad 0.118461 \quad-1.585051 \quad 0.188060$
$\begin{array}{llll}\text { C } & 0.556066 & -1.332625 & -2.545904\end{array}$
$\begin{array}{llll}\text { C } & 1.162600 & -2.508828 & 0.288289\end{array}$
O $\quad 3.240590 \quad-2.877716 \quad-0.821394$ 

H $\quad 0.825933 \quad-0.958173 \quad-3.543392$
C $\quad-0.038073 \quad-0.134123 \quad-1.808069$
$\begin{array}{llll}\text { O } & -1.240864 & 0.000392 & -1.673748\end{array}$
$\begin{array}{llll}\text { C } & 0.711678 & -3.958690 & 0.474078\end{array}$
H $\quad 1.586452 \quad-4.602624 \quad 0.587748$

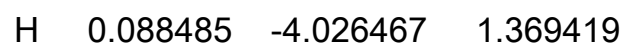
$\begin{array}{llll}\mathrm{H} & 0.125341 & -4.303835 & -0.383020\end{array}$
H $\quad 1.793648 \quad-2.230142 \quad 1.141711$
$\begin{array}{llll}\text { C } & -4.265317 & -0.298455 & -0.670230\end{array}$
$\begin{array}{llll}\text { C } & -4.269248 & 0.970914 & -1.181993\end{array}$
$\begin{array}{llll}N & -4.653829 & -0.543743 & 0.593457\end{array}$
$\begin{array}{llll}\text { C } & -4.624158 & 2.068161 & -0.351694\end{array}$
H $\quad-3.896020 \quad 1.115578-2.185119$
$\begin{array}{llll}\text { C } & -5.052551 & 0.459987 & 1.395230\end{array}$
H $\quad-4.564001 \quad-1.524578 \quad 0.967386$
$\begin{array}{llll}C & -5.068187 & 1.757134 & 0.963418\end{array}$
$\begin{array}{llll}N & -4.509661 & 3.342664 & -0.774344\end{array}$
$\begin{array}{llll}\mathrm{H} & -5.331516 & 0.180360 & 2.403455\end{array}$
H $\quad-5.347102 \quad 2.531024 \quad 1.663278$
$\begin{array}{llll}C & -3.898326 & 3.625824 & -2.066640\end{array}$
$\begin{array}{llll}\text { C } & -4.735409 & 4.441629 & 0.158129\end{array}$
H $\quad-4.462551 \quad 3.153300 \quad-2.877166$
H $\quad-3.912158 \quad 4.701718-2.233220$
$\begin{array}{llll}H & -2.855859 & 3.288281 & -2.086827\end{array}$
$\begin{array}{llll}H & -4.041414 & 4.382508 & 1.003909\end{array}$
H $\quad-4.568227 \quad 5.382955 \quad-0.362802$
$\begin{array}{llll}\mathrm{H} & -5.768663 & 4.437116 & 0.521437\end{array}$
$\begin{array}{llll}\text { O } & -4.326176 & -3.032496 & 1.461964\end{array}$
S $\quad-2.942566 \quad-3.532894 \quad 1.190876$
$\begin{array}{llll}\text { O } & -2.081570 & -3.617387 & 2.373910\end{array}$
$\begin{array}{llll}\mathrm{O} & -2.327715 & -2.774468 & 0.054572\end{array}$
C $\quad-3.169221 \quad-5.200263 \quad 0.587661$
H $\quad-3.653942 \quad-5.780526 \quad 1.373180$
H $\quad-3.791242 \quad-5.168483 \quad-0.307076$
H $\quad-2.185369-5.613590 \quad 0.361162$
H $\quad-0.758284 \quad-2.022295 \quad 0.263533$
$\begin{array}{llll}\mathrm{O} & -1.838684 & 1.155377 & 0.681157\end{array}$
H $\quad-3.899375 \quad-1.155978 \quad-1.221637$
H $\quad-1.579933 \quad 0.810786 \quad-0.215259$
O $\quad 0.743676 \quad 0.867280 \quad-1.406523$ 


\begin{tabular}{|c|c|c|c|}
\hline C & 2.105317 & 1.061994 & -1.802038 \\
\hline $\mathrm{H}$ & 2.309602 & 0.573606 & -2.756643 \\
\hline S & -1.254288 & 2.606394 & 0.955269 \\
\hline c & -2.138477 & 3.216125 & 1.937609 \\
\hline & 1.829981 & -1.740265 & -2.039397 \\
\hline & -0.436082 & -2.466738 & -2.732430 \\
\hline & -1.242453 & -2.134786 & -3.390132 \\
\hline & 0.078140 & -3.305433 & -3.208653 \\
\hline H & -0.888269 & -2.782523 & -1.792317 \\
\hline C & 0.306341 & 2.245390 & 1.721102 \\
\hline H & 0.894943 & 1.610058 & 1.056426 \\
\hline $\mathrm{H}$ & 0.805645 & 3.197006 & 1.908873 \\
\hline & 0.099477 & 1.725358 & 2.656371 \\
\hline C & -1.037627 & 3.291983 & -0.313805 \\
\hline & 2.315880 & 2.566488 & -1.907550 \\
\hline $\mathrm{H}$ & 2.059351 & 3.046739 & -0.960966 \\
\hline $\mathrm{H}$ & 3.361731 & 2.777643 & -2.141740 \\
\hline & 1.672814 & 2.975681 & -2.689077 \\
\hline C & 5.209068 & -0.320728 & -0.395770 \\
\hline $\mathrm{H}$ & 4.759087 & -1.060966 & 0.270539 \\
\hline C & 6.294373 & -0.962406 & -1.243368 \\
\hline r & 5.854755 & -1.784478 & -1.811325 \\
\hline H & 6.729992 & -0.234598 & -1.933235 \\
\hline $\mathrm{H}$ & 7.079502 & -1.358363 & -0.596109 \\
\hline C & 7.033394 & 1.368842 & 2.345958 \\
\hline $\mathrm{H}$ & 7.349416 & 2.243310 & 1.772408 \\
\hline $\mathrm{H}$ & 7.908459 & 0.878036 & 2.774607 \\
\hline O & 4.209085 & 0.170961 & -1.290416 \\
\hline $\mathrm{O}$ & 6.488887 & 0.389515 & 1.437518 \\
\hline C & 3.033176 & 0.478814 & -0.748662 \\
\hline C & 5.726091 & 0.843935 & 0.441409 \\
\hline O & 2.749305 & 0.345713 & 0.418622 \\
\hline O & 5.493587 & 2.009392 & 0.220764 \\
\hline C & 6.020985 & 1.740262 & 3.412794 \\
\hline H & 5.149475 & 2.224292 & 2.966252 \\
\hline & 5.692914 & 0.853056 & 3.960538 \\
\hline & 6.476820 & 2.436979 & 4.122784 \\
\hline
\end{tabular}


For DMAP:MSA (1:2) - Initiation

\section{Complex 11}

\begin{tabular}{lrrr}
$\mathrm{C}$ & 3.801527 & 1.730157 & 0.326266 \\
$\mathrm{O}$ & 2.119502 & 0.285783 & 1.308150 \\
$\mathrm{C}$ & 3.805213 & -0.610356 & -0.158495 \\
$\mathrm{C}$ & 2.310317 & 1.500576 & 0.538481 \\
$\mathrm{O}$ & 4.333419 & 2.807299 & 0.364294 \\
$\mathrm{H}$ & 3.242009 & -0.527147 & -1.096611 \\
$\mathrm{C}$ & 2.759686 & -0.812096 & 0.920258 \\
$\mathrm{O}$ & 2.490996 & -1.880731 & 1.432891 \\
$\mathrm{C}$ & 1.619443 & 2.619744 & 1.278227 \\
$\mathrm{H}$ & 1.771283 & 3.553592 & 0.734832 \\
$\mathrm{H}$ & 0.550038 & 2.404099 & 1.314188 \\
$\mathrm{H}$ & 2.030970 & 2.733475 & 2.284196 \\
$\mathrm{H}$ & 1.826669 & 1.352268 & -0.434937 \\
$\mathrm{C}$ & -1.005841 & -2.242422 & 0.686811 \\
$\mathrm{C}$ & -2.307842 & -1.942808 & 0.418784 \\
$\mathrm{~N}$ & -0.312308 & -1.575107 & 1.632588 \\
$\mathrm{C}$ & -2.960381 & -0.892176 & 1.129322 \\
$\mathrm{H}$ & -2.804026 & -2.472222 & -0.380600 \\
$\mathrm{C}$ & -0.881404 & -0.560695 & 2.314954 \\
$\mathrm{H}$ & 0.692442 & -1.760008 & 1.734255 \\
$\mathrm{C}$ & -2.183954 & -0.210904 & 2.112075 \\
$\mathrm{~N}$ & -4.232870 & -0.559502 & 0.878029 \\
$\mathrm{H}$ & -0.235813 & -0.031035 & 3.003376 \\
$\mathrm{H}$ & -2.576735 & 0.643599 & 2.642311 \\
$\mathrm{C}$ & -4.908964 & -1.112539 & -0.291689 \\
$\mathrm{C}$ & -4.845387 & 0.568248 & 1.571495 \\
$\mathrm{H}$ & -5.033256 & -2.196528 & -0.194047 \\
$\mathrm{H}$ & -5.899314 & -0.666478 & -0.365579 \\
$\mathrm{H}$ & -4.341784 & -0.869961 & -1.196891 \\
-4.780657 & 0.431047 & 2.655312 \\
$\mathrm{H}$ & -4.358957 & 1.502243 & 1.271241 \\
\hline
\end{tabular}




$\begin{array}{lrrr}\mathrm{H} & -2.593777 & 2.883893 & -2.928297 \\ \mathrm{H} & -1.177794 & 3.536362 & -2.044751 \\ \mathrm{H} & -0.958715 & 2.225659 & -3.251410 \\ \mathrm{H} & 0.317680 & -0.248323 & -1.081199 \\ \mathrm{H} & -0.456521 & -2.999911 & 0.144150 \\ \mathrm{O} & 0.940573 & -0.993270 & -1.250208 \\ \mathrm{C} & 0.527144 & -1.691903 & -2.407663 \\ \mathrm{H} & 0.538494 & -1.027995 & -3.283631 \\ \mathrm{H} & -0.508978 & -2.041143 & -2.290587 \\ \mathrm{C} & 1.468381 & -2.862830 & -2.624505 \\ \mathrm{H} & 1.170767 & -3.444757 & -3.502029 \\ \mathrm{H} & 2.493566 & -2.510411 & -2.779798 \\ \mathrm{H} & 1.468411 & -3.524872 & -1.751925 \\ \mathrm{O} & 4.519394 & 0.616350 & 0.080218 \\ \mathrm{C} & 4.811939 & -1.734596 & -0.234364 \\ \mathrm{H} & 4.293482 & -2.671409 & -0.446061 \\ \mathrm{H} & 5.525448 & -1.528759 & -1.034471 \\ \mathrm{H} & 5.352080 & -1.837821 & 0.709190\end{array}$

TS $/ 1$
C $\quad-3.857765 \quad-0.117396-1.200025$
$\begin{array}{lllll}\mathrm{O} & -1.496996 & -0.820243 & -0.748494\end{array}$
$\begin{array}{llll}\text { C } & -3.311580 & -1.608045 & 0.622621\end{array}$
$\begin{array}{llll}\text { C } & -2.378570 & -0.029175 & -1.562476\end{array}$
$\begin{array}{llll}\text { O } & -4.682930 & 0.464590 & -1.858585\end{array}$
$\begin{array}{llll}\mathrm{H} & -3.660907 & -1.560876 & 1.656386\end{array}$
$\begin{array}{llll}\text { C } & -1.888320 & -1.058850 & 0.558418\end{array}$
$\begin{array}{llll}\mathrm{O} & -1.036834 & -1.683524 & 1.270506\end{array}$
$\begin{array}{llll}\text { C } & -2.153451 & -0.444193 & -3.007719\end{array}$
H $\quad-2.776107 \quad 0.166379 \quad-3.662988$
H $\quad-1.104631 \quad-0.290289-3.272672$
H $\quad-2.403418-1.499208 \quad-3.154568$
H $\quad-2.109731 \quad 1.027465 \quad-1.434273$
$\begin{array}{llll}\text { C } & 2.139471 & -0.397577 & 1.544465\end{array}$
C $3.457233 \quad-0.138670 \quad 1.261668$
N $\quad 1.439486 \quad-1.320973 \quad 0.866206$
$\begin{array}{llll}\text { C } & 4.109129 & -0.846523 & 0.219600\end{array}$
$\begin{array}{llll}\mathrm{H} & 3.956883 & 0.637543 & 1.823345\end{array}$ 


\begin{tabular}{llll}
$\mathrm{C}$ & 2.024715 & -2.018311 & -0.118136 \\
$\mathrm{H}$ & 0.353230 & -1.448039 & 1.045118 \\
$\mathrm{C}$ & 3.339333 & -1.826013 & -0.464512 \\
$\mathrm{~N}$ & 5.393891 & -0.588416 & -0.116859 \\
$\mathrm{H}$ & 1.388751 & -2.729129 & -0.633198 \\
$\mathrm{H}$ & 3.752271 & -2.410438 & -1.274803 \\
$\mathrm{C}$ & 6.119439 & 0.484714 & 0.549154 \\
$\mathrm{C}$ & 6.025431 & -1.317283 & -1.205328 \\
$\mathrm{H}$ & 6.233243 & 0.281812 & 1.619746 \\
$\mathrm{H}$ & 7.114131 & 0.564969 & 0.113699 \\
$\mathrm{H}$ & 5.611660 & 1.446991 & 0.420910 \\
$\mathrm{H}$ & 6.026203 & -2.395448 & -1.011572 \\
$\mathrm{H}$ & 5.522969 & -1.128332 & -2.161536 \\
$\mathrm{H}$ & 7.061074 & -0.994051 & -1.297129 \\
$\mathrm{O}$ & 1.539253 & 3.311523 & -0.051964 \\
$\mathrm{~S}$ & 0.489680 & 2.349437 & -0.380841 \\
$\mathrm{O}$ & -0.730985 & 2.841755 & -1.027923 \\
$\mathrm{O}$ & 0.137786 & 1.503075 & 0.849656 \\
$\mathrm{C}$ & 1.214868 & 1.162954 & -1.512085 \\
$\mathrm{H}$ & 1.371021 & 1.670975 & -2.464673 \\
$\mathrm{H}$ & 2.168395 & 0.828779 & -1.102453 \\
$\mathrm{H}$ & 0.525459 & 0.324809 & -1.621866 \\
$\mathrm{H}$ & -1.054074 & 0.983234 & 0.906987 \\
$\mathrm{H}$ & 1.594773 & 0.157208 & 2.295609 \\
$\mathrm{O}$ & -2.040435 & 0.505922 & 1.089139 \\
$\mathrm{C}$ & -2.397687 & 0.699343 & 2.467536 \\
$\mathrm{H}$ & -1.666296 & 1.397600 & 2.885008 \\
$\mathrm{H}$ & -2.279248 & -0.257726 & 2.987875 \\
$\mathrm{C}$ & -3.803782 & 1.256819 & 2.574738 \\
$\mathrm{H}$ & -4.062708 & 1.408536 & 3.627339 \\
\hline & -3.870915 & 2.216781 & 2.057092 \\
\hline & -3.352299 & -3.046398 & 0.129291 \\
\hline & -3.581200 & 2.128483 \\
\hline
\end{tabular}

Int. $1 a$ 


\begin{tabular}{lrrr}
$\mathrm{C}$ & 3.721762 & 1.024167 & -0.970199 \\
$\mathrm{O}$ & 1.571857 & 0.570567 & 0.188292 \\
$\mathrm{C}$ & 3.618336 & -0.250475 & 1.087371 \\
$\mathrm{C}$ & 2.202901 & 1.206827 & -0.927631 \\
$\mathrm{O}$ & 4.358226 & 1.486221 & -1.884717 \\
$\mathrm{H}$ & 4.172145 & -1.166251 & 1.311028 \\
$\mathrm{C}$ & 2.210931 & -0.631230 & 0.612106 \\
$\mathrm{O}$ & 1.533184 & -1.172182 & 1.646488 \\
$\mathrm{C}$ & 1.855625 & 2.687535 & -0.893110 \\
$\mathrm{H}$ & 2.372498 & 3.198688 & -1.707023 \\
$\mathrm{H}$ & 0.779773 & 2.808685 & -1.032107 \\
$\mathrm{H}$ & 2.164657 & 3.131127 & 0.058763 \\
$\mathrm{H}$ & 1.826110 & 0.755134 & -1.850775 \\
$\mathrm{C}$ & -1.844733 & -2.119272 & 1.481412 \\
$\mathrm{C}$ & -3.129063 & -1.824374 & 1.072317 \\
$\mathrm{~N}$ & -0.770021 & -2.076333 & 0.682091 \\
$\mathrm{C}$ & -3.360103 & -1.428690 & -0.264282 \\
$\mathrm{H}$ & -2.217736 & 4.289810 & 0.329615 \\
$\mathrm{H}$ & -3.925999 & -1.847913 & 1.803156 \\
$\mathrm{C}$ & -0.991615 & -1.745550 & -0.598466 \\
$\mathrm{H}$ & -0.681061 & -1.599977 & 1.303308 \\
$\mathrm{C}$ & -2.231930 & -1.430546 & -1.114420 \\
$\mathrm{~N}$ & -4.594516 & -1.046546 & -0.704341 \\
$\mathrm{H}$ & -0.106760 & -1.688700 & -1.227174 \\
$\mathrm{H}$ & -2.299475 & -1.128391 & -2.150405 \\
$\mathrm{C}$ & -5.643935 & -0.808074 & 0.271665 \\
$\mathrm{C}$ & -4.701948 & -0.382463 & -1.993169 \\
$\mathrm{H}$ & -5.367045 & -0.012875 & 0.976205 \\
$\mathrm{H}$ & -6.553344 & -0.511625 & -0.251306 \\
$\mathrm{H}$ & -5.870987 & -1.722078 & 0.829275 \\
\hline & -4.389039 & -1.049625 & -2.802458 \\
$\mathrm{H}$ & -3.0976903 & -0.120460 & -2.170403 \\
\hline
\end{tabular}




$\begin{array}{lrrr}\mathrm{H} & -0.047600 & 0.887727 & 0.713413 \\ \mathrm{H} & -1.655377 & -2.376977 & 2.520066 \\ \mathrm{O} & 2.225241 & -1.434261 & -0.538737 \\ \mathrm{C} & 2.778110 & -2.748518 & -0.416469 \\ \mathrm{H} & 2.024379 & -3.433996 & -0.816390 \\ \mathrm{H} & 2.909939 & -2.998450 & 0.641404 \\ \mathrm{C} & 4.078598 & -2.858990 & -1.192804 \\ \mathrm{H} & 4.462576 & -3.882499 & -1.135552 \\ \mathrm{H} & 3.917184 & -2.608702 & -2.244763 \\ \mathrm{H} & 4.838114 & -2.178134 & -0.798941 \\ \mathrm{O} & 4.349028 & 0.358093 & 0.008210 \\ \mathrm{C} & 3.616507 & 0.664554 & 2.297476 \\ \mathrm{H} & 3.147170 & 0.157706 & 3.142551 \\ \mathrm{H} & 4.643856 & 0.929359 & 2.557598 \\ \mathrm{H} & 3.055564 & 1.578891 & 2.086450\end{array}$

TS 12
C $\quad 3.574265 \quad 1.011145 \quad-0.958314$
$\begin{array}{lllll}\text { O } & 1.656099 & 0.673516 & 0.492309\end{array}$
$\begin{array}{llll}\text { C } & 3.714968 & -0.388117 & 1.057779\end{array}$
$\begin{array}{llll}\text { C } & 2.111591 & 1.367935 & -0.703988\end{array}$
O $4.120309 \quad 1.405564 \quad-1.959512$
H $4.318682 \quad-1.300316 \quad 1.131524$
$\begin{array}{llll}\text { C } & 2.295980 & -0.878841 & 0.790964\end{array}$
$\begin{array}{llll}\text { O } & 1.665130 & -1.426302 & 1.715647\end{array}$
$\begin{array}{llll}\text { C } & 1.958670 & 2.879292 & -0.587281\end{array}$
H $\quad 2.432284 \quad 3.354225 \quad-1.448397$
H $\quad 0.898118 \quad 3.132585 \quad-0.596422$
H $2.428085 \quad 3.2447180 .330783$
H $\quad 1.547409 \quad 1.002769-1.567000$
$\begin{array}{llll}\text { C } & -1.848288 & -1.942936 & 1.612627\end{array}$
$\begin{array}{llll}\text { C } & -3.124293 & -1.728585 & 1.172794\end{array}$
$\begin{array}{llll}N & -0.798530 & -1.911200 & 0.772761\end{array}$
$\begin{array}{llll}\text { C } & -3.356996 & -1.401036 & -0.191579\end{array}$
H $\quad-3.922630 \quad-1.724665 \quad 1.900031$
C $\quad-0.983879 \quad-1.654209 \quad-0.535935$
H $\quad 0.177291 \quad-1.861295 \quad 1.162927$
$\begin{array}{llll}\text { C } & -2.227754 & -1.432310 & -1.053451\end{array}$ 


\begin{tabular}{llll}
$\mathrm{N}$ & -4.577444 & -1.047598 & -0.641147 \\
$\mathrm{H}$ & -0.078891 & -1.576654 & -1.126607 \\
$\mathrm{H}$ & -2.304916 & -1.174199 & -2.098532 \\
$\mathrm{C}$ & -5.630011 & -0.756226 & 0.323168 \\
$\mathrm{C}$ & -4.691628 & -0.435927 & -1.960934 \\
$\mathrm{H}$ & -5.344876 & 0.087274 & 0.963563 \\
$\mathrm{H}$ & -6.540899 & -0.500121 & -0.216457 \\
$\mathrm{H}$ & -5.848828 & -1.634331 & 0.937271 \\
$\mathrm{H}$ & -4.376326 & -1.134757 & -2.740930 \\
$\mathrm{H}$ & -5.736187 & -0.189142 & -2.144281 \\
$\mathrm{H}$ & -4.092000 & 0.480507 & -2.011318 \\
$\mathrm{O}$ & -3.189713 & 1.415977 & 0.275401 \\
$\mathrm{~S}$ & -1.801929 & 1.892253 & 0.150602 \\
$\mathrm{O}$ & -1.297028 & 2.021650 & -1.227005 \\
$\mathrm{O}$ & -0.849949 & 1.082640 & 1.007332 \\
$\mathrm{C}$ & -1.782583 & 3.526126 & 0.881724 \\
$\mathrm{H}$ & -2.438598 & 4.165336 & 0.289817 \\
$\mathrm{H}$ & -2.147416 & 3.444386 & 1.905956 \\
$\mathrm{H}$ & -0.762142 & 3.909643 & 0.870275 \\
$\mathrm{H}$ & 0.561855 & 0.849650 & 0.715008 \\
$\mathrm{H}$ & -1.617051 & -2.113315 & 2.656539 \\
$\mathrm{O}$ & 2.120628 & -1.371502 & -0.517596 \\
$\mathrm{C}$ & 2.644138 & -2.687527 & -0.747007 \\
$\mathrm{H}$ & 1.839896 & -3.261995 & -1.220661 \\
$\mathrm{H}$ & 2.862442 & -3.168405 & 0.212996 \\
$\mathrm{C}$ & 3.864580 & -2.637394 & -1.649347 \\
$\mathrm{H}$ & 4.207852 & -3.653864 & -1.868095 \\
$\mathrm{H}$ & 3.621101 & -2.142118 & -2.593025 \\
$\mathrm{H}$ & 4.683707 & -2.083272 & -1.184140 \\
$\mathrm{O}$ & 4.285962 & 0.306973 & -0.062979 \\
\hline & 3.863959 & 0.419893 & 2.331811 \\
\hline & 3.279549 & 1.340145 & 2.271877
\end{tabular}

Complex 12

$\begin{array}{llll}C & 2.696005 & 2.168279 & 0.023730\end{array}$

$\begin{array}{llll}\text { O } & 1.300669 & 0.283931 & -0.731723\end{array}$ 


\begin{tabular}{lrrr}
$\mathrm{C}$ & 2.965873 & 0.200733 & 1.580990 \\
$\mathrm{C}$ & 2.031929 & 1.413953 & -1.140149 \\
$\mathrm{O}$ & 2.872892 & 3.359785 & -0.014362 \\
$\mathrm{H}$ & 3.818467 & 0.031822 & 2.253277 \\
$\mathrm{C}$ & 3.077022 & -0.943785 & 0.583994 \\
$\mathrm{O}$ & 2.511512 & -2.017061 & 0.731245 \\
$\mathrm{C}$ & 1.205823 & 2.351604 & -2.009127 \\
$\mathrm{H}$ & 1.811311 & 3.184997 & -2.368088 \\
$\mathrm{H}$ & 0.810697 & 1.788971 & -2.858078 \\
$\mathrm{H}$ & 0.360280 & 2.751551 & -1.446362 \\
$\mathrm{H}$ & 2.878955 & 1.034886 & -1.726264 \\
$\mathrm{C}$ & -1.182455 & -2.068433 & 1.453896 \\
$\mathrm{C}$ & -2.509950 & -1.894796 & 1.199007 \\
$\mathrm{~N}$ & -0.271702 & -2.093846 & 0.458646 \\
$\mathrm{C}$ & -2.957034 & -1.684119 & -0.138614 \\
$\mathrm{H}$ & -3.187781 & -1.821323 & 2.035832 \\
$\mathrm{C}$ & -0.653712 & -1.927253 & -0.825193 \\
$\mathrm{H}$ & -1.262734 & 3.806069 & -0.219121 \\
$\mathrm{H}$ & 0.731226 & -2.125323 & 0.674094 \\
$\mathrm{C}$ & -1.962007 & -1.739589 & -1.157385 \\
$\mathrm{~N}$ & -4.240578 & -1.428825 & -0.424950 \\
$\mathrm{H}$ & 0.143647 & -1.900462 & -1.555084 \\
$\mathrm{H}$ & -2.205439 & -1.548604 & -2.191510 \\
$\mathrm{C}$ & -5.204669 & -1.249132 & 0.654751 \\
$\mathrm{C}$ & -4.603458 & -0.972585 & -1.764694 \\
$\mathrm{H}$ & -4.945154 & -0.363045 & 1.244108 \\
$\mathrm{H}$ & -6.194705 & -1.117107 & 0.220675 \\
$\mathrm{H}$ & -5.237727 & -2.138296 & 1.291833 \\
$\mathrm{H}$ & -4.409169 & -1.754245 & -2.506632 \\
$\mathrm{H}$ & -5.669931 & -0.754184 & -1.781263 \\
\hline & -4.047112 & -0.061048 & -2.012149 \\
\hline & -266188 & 1.182908 & 1.054470 \\
\hline
\end{tabular}




$\begin{array}{llll}\mathrm{O} & 3.977386 & -0.712876 & -0.357236 \\ \mathrm{C} & 4.179046 & -1.735622 & -1.353290 \\ \mathrm{H} & 4.547838 & -1.188263 & -2.222393 \\ \mathrm{H} & 3.211779 & -2.183822 & -1.591585 \\ \mathrm{C} & 5.179741 & -2.773481 & -0.883831 \\ \mathrm{H} & 5.366261 & -3.489949 & -1.689401 \\ \mathrm{H} & 6.128930 & -2.301062 & -0.617665 \\ \mathrm{H} & 4.796233 & -3.319529 & -0.019215 \\ \mathrm{O} & 3.246080 & 1.499899 & 1.071245 \\ \mathrm{C} & 1.695833 & 0.172675 & 2.414994 \\ \mathrm{H} & 1.607421 & -0.797400 & 2.911757 \\ \mathrm{H} & 1.768082 & 0.946445 & 3.182117 \\ \mathrm{H} & 0.802036 & 0.363535 & 1.818603\end{array}$


For DMAP:MSA (1:2) - Propagation

\section{Complex P1}

\begin{tabular}{|c|c|c|c|}
\hline & & & \\
\hline & -0.281577 & -1.513025 & 71690 \\
\hline & -0.377390 & -2.189011 & -0.55 \\
\hline & 1.156260 & & \\
\hline & 2.5 & & \\
\hline & 0.134816 & & -1 \\
\hline & -1.058416 & & \\
\hline & -2.252900 & & \\
\hline & 1.824609 & & \\
\hline & 2.904941 & & \\
\hline & 1.51 & -0.5 & \\
\hline & 1.570058 & -2.357 & \\
\hline & 1.40 & & \\
\hline & -3.83 & & \\
\hline & -5.0 & & \\
\hline & -2.7 & & \\
\hline & -5.201066 & & \\
\hline & -5.942106 & 2.807665 & -0.8 \\
\hline & -2.786187 & 1.672398 & \\
\hline & -1.769955 & & \\
\hline & -3.99 & & \\
\hline & -6.4 & & \\
\hline & -1.834691 & & \\
\hline & -3.990145 & 3761 & \\
\hline & -7.616924 & & \\
\hline & -6.495061 & & \\
\hline & -7.57 & & \\
\hline & -8.47 & & \\
\hline & -7.760910 & & \\
\hline & -6.089323 & -0.380121 & 2.443 \\
\hline & -5.964133 & & \\
\hline & -7.542285 & & \\
\hline & -0.256019 & 3.060974 & -1.0 \\
\hline & 0.735798 & 2.020500 & -0.6 \\
\hline & 1.596607 & 1.568926 & \\
\hline & & & \\
\hline & & & \\
\hline
\end{tabular}




\begin{tabular}{|c|c|c|c|}
\hline & 2.284057 & 3.662168 & \\
\hline & 1.221820 & 3.117581 & 1389758 \\
\hline & 2.570355 & 2.065383 & 0025211 \\
\hline & 2.084313 & -0.107241 & \\
\hline & -3.934567 & -0.739358 & 5926 \\
\hline & -3.690896 & 3.349731 & -1.728518 \\
\hline & -3.319424 & -1.057915 & -0.32778 \\
\hline & 2.263110 & -1.068769 & -1.5 \\
\hline & 3.640629 & -1.296240 & -1.5 \\
\hline & 3.788506 & -2.380741 & -1.5 \\
\hline & -5.400004 & -1.325898 & -0.9 \\
\hline & -6.284645 & -0.408456 & -1. \\
\hline & 0.612072 & -3.161518 & -0 \\
\hline & -1.313108 & -2.854579 & -1.5 \\
\hline & -2.021025 & -2.1 & \\
\hline & -0.721367 & -3.251213 & -2.3 \\
\hline & -1.860592 & -3.675178 & 6788 \\
\hline & -5.297285 & -2.838474 & -1.85410 \\
\hline & -4.562716 & -3.488177 & $-1.37747 \varepsilon$ \\
\hline & -6.285505 & & \\
\hline & -5.003376 & -2.592 & \\
\hline & -5.665413 & -1.6 & \\
\hline & 6.231257 & -0.7 & 1. \\
\hline & 5.649923 & -0.803123 & 1.940476 \\
\hline & 4.358707 & -0.642334 & -2.7 \\
\hline & 4.208986 & 0.441694 & -2.709717 \\
\hline & 5.430602 & -0.851391 & \\
\hline & 3.932349 & -1.031268 & -3.65 \\
\hline & 7.531055 & -1.505070 & 1.125 \\
\hline & 7.311112 & -2.557116 & 1.318707 \\
\hline & 8.102849 & -1.429013 & 0.196965 \\
\hline & 8.129583 & -1.108900 & \\
\hline & 6.530171 & 2.5135 & -0.79 \\
\hline & 7.305218 & 2.961 & -0.168 \\
\hline & 6.849738 & 2.52 & -1.8 \\
\hline & 5.190518 & 3.199982 & 610051 \\
\hline & 4.901586 & 3.194604 & \\
\hline & 5.260689 & 4.239727 & \\
\hline & 4.411431 & 2.698312 & -1.191290 \\
\hline & 4.254238 & -0.790722 & -0.24768 \\
\hline
\end{tabular}



C $\quad 6.499124$
0.764129
0.805905
O $5.488353 \quad-1.282053 \quad-0.065239$
$\begin{array}{llll}\text { O } & 6.423278 & 1.112013 & -0.478356\end{array}$
$\begin{array}{llll}\text { O } & 6.790706 & 1.506468 & 1.715167\end{array}$
$\begin{array}{llll}\text { O } & 3.733380 & -0.008567 & 0.518594\end{array}$

TS P1
C $\quad-0.053885 \quad-4.249565 \quad-0.664764$
$\begin{array}{llll}\mathrm{O} & -1.226914 & -2.195076 & 0.057653\end{array}$
C $\quad-0.763396-2.543198 \quad-2.245890$
$\begin{array}{llll}\text { C } & -0.597928 & -3.403312 & 0.487430\end{array}$
O $0.459725 \quad-5.318182 \quad-0.448991$
H $\quad-0.216780 \quad-2.160605 \quad-3.110971$
$\begin{array}{llll}\text { C } & -0.605929 & -1.571346 & -1.069821\end{array}$
$\begin{array}{llll}\mathrm{O} & -1.263084 & -0.425573 & -1.339897\end{array}$
$\begin{array}{llll}\text { C } & -1.601319 & -4.192688 & 1.312218\end{array}$
$\begin{array}{llll}\mathrm{H} & -1.128652 & -5.101998 & 1.685669\end{array}$

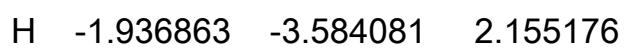
$\begin{array}{llll}H & -2.464290 & -4.475915 & 0.699367\end{array}$
H $\quad 0.272977 \quad-3.138759 \quad 1.096053$
C $\quad-3.682870 \quad 1.547232 \quad-1.017382$
$\begin{array}{llll}\text { C } & -2.931729 & 2.461380 & -1.696894\end{array}$
$\begin{array}{llll}N & -4.136065 & 1.799827 & 0.226598\end{array}$
$\begin{array}{llll}\text { C } & -2.565352 & 3.687715 & -1.071757\end{array}$
H $\quad-2.542023 \quad 2.181088 \quad-2.664067$
$\begin{array}{llll}\text { C } & -3.839161 & 2.962193 & 0.846354\end{array}$
H $\quad-4.625691 \quad 1.065259 \quad 0.745034$
$\begin{array}{llll}C & -3.091097 & 3.920214 & 0.232876\end{array}$
$\begin{array}{llll}\mathrm{N} & -1.746087 & 4.569147 & -1.663166\end{array}$
$\begin{array}{llll}H & -4.202023 & 3.069227 & 1.860107\end{array}$
H $\quad-2.829038 \quad 4.803655 \quad 0.794451$
C $\quad-1.097979 \quad 4.220619 \quad-2.921506$
C $\quad-1.202123 \quad 5.682015 \quad-0.887548$
$\begin{array}{llll}H & -1.843707 & 3.987942 & -3.687810\end{array}$
H $\quad-0.523024 \quad 5.078008 \quad-3.268173$
H $\quad-0.418962 \quad 3.373931 \quad-2.770465$
$\begin{array}{llll}H & -0.678170 & 5.305225 & -0.001788\end{array}$
H $\quad-0.496399 \quad 6.228075 \quad-1.511097$ 


\begin{tabular}{|c|c|c|c|}
\hline $\mathrm{H}$ & -1.998678 & 6.373655 & -0.593351 \\
\hline 0 & -5.272965 & -0.342798 & 1.712462 \\
\hline$S$ & -4.392754 & -1.512228 & 1.732170 \\
\hline 0 & -3.380175 & -1.631397 & 2.761622 \\
\hline 0 & -3.739719 & -1.589760 & $0.29027 \mathrm{c}$ \\
\hline C & -5.428786 & -2.956508 & 1.749613 \\
\hline $\mathrm{H}$ & -5.980973 & -2.943991 & 2.689950 \\
\hline $\mathrm{H}$ & -6.110037 & -2.906765 & 0.900698 \\
\hline $\mathrm{H}$ & -4.787597 & -3.836350 & 1.692605 \\
\hline $\mathrm{H}$ & -2.750899 & -1.789630 & 0.300093 \\
\hline $\mathrm{O}$ & -1.030810 & 1.234462 & 0.622613 \\
\hline $\mathrm{H}$ & -3.907284 & 0.566637 & -1.416142 \\
\hline $\mathrm{H}$ & -1.109236 & 0.244017 & -0.593941 \\
\hline 0 & 0.717412 & -1.398694 & -0.655314 \\
\hline C & 1.759414 & -1.251091 & -1.603540 \\
\hline $\mathrm{H}$ & 1.803844 & -2.135009 & -2.256725 \\
\hline$S$ & 0.058739 & 2.270951 & 0.609588 \\
\hline $\mathrm{O}$ & -0.294487 & 3.463479 & 1.403454 \\
\hline $\mathrm{O}$ & -0.117348 & -3.788967 & -1.92746 \\
\hline C & -2.209983 & -2.789868 & -2.632643 \\
\hline $\mathrm{H}$ & -2.672811 & -1.853611 & -2.950147 \\
\hline $\mathrm{H}$ & -2.247864 & -3.508437 & -3.454403 \\
\hline $\mathrm{H}$ & -2.778756 & -3.189500 & -1.788982 \\
\hline C & 1.446641 & 1.498557 & 1.429626 \\
\hline $\mathrm{H}$ & 1.639287 & 0.531753 & 0.963236 \\
\hline $\mathrm{H}$ & 2.309180 & 2.157553 & 1.317921 \\
\hline $\mathrm{H}$ & 1.181948 & 1.362766 & 2.479400 \\
\hline $\mathrm{O}$ & 0.499443 & 2.574446 & -0.771322 \\
\hline C & 1.664218 & 0.024716 & -2.436562 \\
\hline $\mathrm{H}$ & 1.596597 & 0.907234 & -1.795146 \\
\hline $\mathrm{H}$ & 2.541148 & 0.103957 & -3.082138 \\
\hline $\mathrm{H}$ & 0.765461 & -0.000712 & -3.057352 \\
\hline C & 5.361672 & -1.073777 & -0.847938 \\
\hline $\mathrm{H}$ & 5.496485 & -1.974780 & -0.244136 \\
\hline C & 6.457468 & -0.933421 & -1.890993 \\
\hline $\mathrm{H}$ & 6.446289 & -1.797687 & -2.558681 \\
\hline $\mathrm{H}$ & 6.306361 & -0.025690 & -2.480416 \\
\hline $\mathrm{H}$ & 7.431867 & -0.882690 & -1.398854 \\
\hline C & 6.098825 & 0.949776 & 2.159961 \\
\hline $\mathrm{H}$ & 6.066599 & 1.937337 & 1.695493 \\
\hline
\end{tabular}




$\begin{array}{lrrr}\mathrm{H} & 7.030470 & 0.827383 & 2.713134 \\ \mathrm{C} & 4.894982 & 0.744371 & 3.071027 \\ \mathrm{H} & 4.930396 & -0.260685 & 3.507572 \\ \mathrm{H} & 3.976558 & 0.825968 & 2.481246 \\ \mathrm{O} & 4.118347 & -1.153013 & -1.547497 \\ \mathrm{O} & 6.114632 & -0.071330 & 1.147203 \\ \mathrm{O} & 4.958338 & 1.722708 & 4.095013 \\ \mathrm{H} & 4.106454 & 2.154824 & 4.179884 \\ \mathrm{C} & 3.032710 & -1.291679 & -0.769346 \\ \mathrm{C} & 5.327883 & 0.133625 & 0.087270 \\ \mathrm{O} & 3.086658 & -1.434388 & 0.427700 \\ \mathrm{O} & 4.700131 & 1.141992 & -0.127909\end{array}$

Int. $P 1$
$\begin{array}{llll}\text { C } & -0.591438 & -3.804783 & 0.298468\end{array}$
$\begin{array}{llll}\mathrm{O} & -1.335885 & -1.641013 & 1.289603\end{array}$

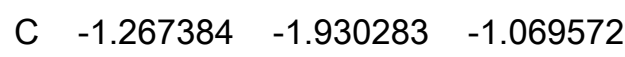
$\begin{array}{llll}\text { C } & -0.892926 & -2.966678 & 1.543544\end{array}$
$\begin{array}{llll}\mathrm{O} & -0.198003 & -4.940366 & 0.412209\end{array}$
H $\quad-0.855729 \quad-1.551621 \quad-2.006371$
C $\quad-0.824880-1.070610 \quad 0.122800$
$\begin{array}{llll}\mathrm{O} & -1.292098 & 0.192805 & 0.112810\end{array}$
$\begin{array}{llll}\text { C } & -1.948345 & -3.642132 & 2.406387\end{array}$

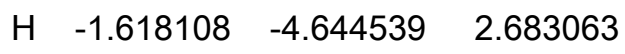
H $\quad-2.105199 \quad-3.050562 \quad 3.311762$
$\begin{array}{llll}H & -2.896507 & -3.719396 & 1.865604\end{array}$
$\begin{array}{llll}H & 0.058349 & -2.926598 & 2.088448\end{array}$
$\begin{array}{llll}\text { C } & -2.549132 & 1.325896 & 2.730276\end{array}$
$\begin{array}{llll}\text { C } & -3.582539 & 0.535123 & 2.321472\end{array}$
$\begin{array}{llll}\mathrm{N} & -2.143668 & 2.378305 & 1.993017\end{array}$
$\begin{array}{llll}\text { C } & -4.209776 & 0.781145 & 1.067758\end{array}$
H $\quad-3.851934 \quad-0.310885 \quad 2.936296$
C $\quad-2.732060 \quad 2.667304 \quad 0.811876$
H $\quad-1.296617 \quad 2.861339 \quad 2.297264$
$\begin{array}{llll}\text { C } & -3.773715 & 1.922932 & 0.340005\end{array}$
$\begin{array}{llll}\mathrm{N} & -5.153167 & -0.044876 & 0.581805\end{array}$
$\begin{array}{llll}\mathrm{H} & -2.304265 & 3.481670 & 0.242639\end{array}$
H $\quad-4.151458 \quad 2.143701 \quad-0.649879$ 


\begin{tabular}{|c|c|c|c|}
\hline C & -5.540052 & -1.227385 & 1.333329 \\
\hline C & -5.819377 & 0.251405 & -0.686137 \\
\hline $\mathrm{H}$ & -6.037398 & -0.965562 & 2.275429 \\
\hline $\mathrm{H}$ & -6.231624 & -1.817895 & 0.734701 \\
\hline $\mathrm{H}$ & -4.666018 & -1.849867 & 1.554329 \\
\hline $\mathrm{H}$ & -6.450586 & 1.143241 & -0.594238 \\
\hline $\mathrm{H}$ & -5.094474 & 0.410060 & -1.490485 \\
\hline $\mathrm{H}$ & -6.453401 & -0.592918 & -0.952796 \\
\hline 0 & 0.439273 & 2.747173 & 3.163286 \\
\hline$S$ & 1.305535 & 1.906115 & 2.331485 \\
\hline $\mathrm{O}$ & 1.439634 & 2.253738 & 0.926813 \\
\hline O & 0.762796 & 0.433750 & 2.500930 \\
\hline C & 2.911220 & 1.824808 & 3.081270 \\
\hline $\mathrm{H}$ & 3.352221 & 2.820057 & 3.015070 \\
\hline $\mathrm{H}$ & 2.788286 & 1.520461 & 4.120357 \\
\hline $\mathrm{H}$ & 3.492685 & 1.093409 & 2.518426 \\
\hline $\mathrm{H}$ & 0.864131 & -0.105356 & 1.663429 \\
\hline O & -1.441141 & 1.754154 & -1.958053 \\
\hline $\mathrm{H}$ & -1.987880 & 1.140315 & 3.636617 \\
\hline $\mathrm{H}$ & -1.254811 & 0.676272 & -0.760521 \\
\hline $\mathrm{O}$ & 0.606212 & -1.098864 & 0.321045 \\
\hline C & 1.512958 & -1.088381 & -0.778741 \\
\hline $\mathrm{H}$ & 1.386641 & -2.007320 & -1.361277 \\
\hline$S$ & -2.238149 & 1.251239 & -3.127155 \\
\hline O & -3.694949 & 1.301982 & -2.866589 \\
\hline O & -0.719902 & -3.258522 & -0.917254 \\
\hline C & -2.777575 & -1.999130 & -1.200669 \\
\hline $\mathrm{H}$ & -3.180097 & -1.005313 & -1.401186 \\
\hline $\mathrm{H}$ & -3.030660 & -2.641741 & -2.045632 \\
\hline $\mathrm{H}$ & -3.230929 & -2.395925 & -0.287779 \\
\hline C & -1.922889 & 2.428042 & -4.435038 \\
\hline $\mathrm{H}$ & -0.852107 & 2.433270 & -4.639971 \\
\hline $\mathrm{H}$ & -2.481171 & 2.107533 & -5.315080 \\
\hline $\mathrm{H}$ & -2.257937 & 3.411509 & -4.104678 \\
\hline $\mathrm{O}$ & -1.742306 & -0.056643 & -3.598147 \\
\hline C & 5.157107 & -1.576243 & -0.563959 \\
\hline $\mathrm{H}$ & 5.255919 & -1.965873 & 0.452259 \\
\hline C & 1.418988 & 0.144777 & -1.678219 \\
\hline $\mathrm{H}$ & 1.451809 & 1.052877 & -1.073630 \\
\hline $\mathrm{H}$ & 2.262176 & 0.138471 & -2.373888 \\
\hline
\end{tabular}




$\begin{array}{lrrr}\text { H } & 0.497420 & 0.144261 & -2.262365 \\ \mathrm{C} & 5.963330 & -2.407602 & -1.549135 \\ \mathrm{H} & 5.618183 & -3.443313 & -1.526193 \\ \mathrm{H} & 5.847521 & -2.019885 & -2.564448 \\ \mathrm{H} & 7.019117 & -2.377698 & -1.271708 \\ \mathrm{C} & 5.265287 & 2.086125 & -1.248896 \\ \mathrm{H} & 6.350790 & 2.166041 & -1.344351 \\ \mathrm{H} & 4.788495 & 2.477968 & -2.148316 \\ \mathrm{C} & 4.755550 & 2.781132 & -0.001623 \\ \mathrm{H} & 5.264285 & 2.395111 & 0.885558 \\ \mathrm{H} & 4.960684 & 3.853671 & -0.074936 \\ \mathrm{H} & 3.676869 & 2.639553 & 0.105979 \\ \mathrm{C} & 2.888566 & -1.119112 & -0.139297 \\ \mathrm{C} & 5.660299 & -0.135943 & -0.527871 \\ \mathrm{O} & 3.786827 & -1.653679 & -0.970065 \\ \mathrm{O} & 4.903018 & 0.688969 & -1.245592 \\ \mathrm{O} & 6.671200 & 0.173057 & 0.060555 \\ \mathrm{O} & 3.158535 & -0.678121 & 0.956110\end{array}$

Int. $P 2$

C $\quad 0.212079-4.177425 \quad-0.698633$

$\begin{array}{llll}\text { O } & -1.071800 & -2.190497 & 0.021903\end{array}$

C $\quad-0.461313 \quad-2.438210 \quad-2.259864$

$\begin{array}{llll}\text { C } & -0.425489 & -3.390557 & 0.447731\end{array}$

O $\quad 0.747209 \quad-5.237013 \quad-0.489830$

H $\quad 0.121868 \quad-2.007084 \quad-3.076924$

$\begin{array}{llll}\text { C } & -0.407000 & -1.505134 & -1.043658\end{array}$

$\begin{array}{lllll}\text { O } & -1.086304 & -0.372537 & -1.312558\end{array}$

$\begin{array}{llll}\text { C } & -1.445137 & -4.240976 & 1.187474\end{array}$

H $\quad-0.962493 \quad-5.146232 \quad 1.558015$

H $\quad-1.849078 \quad-3.673126 \quad 2.028891$

H $\quad-2.261617 \quad-4.531766 \quad 0.517136$

H $\quad 0.400011 \quad-3.117965 \quad 1.113461$

$\begin{array}{llll}\text { C } & -3.591856 & 1.499182 & -1.067731\end{array}$

C $\quad-2.824024 \quad 2.451918-1.671370$

N $\quad-4.125719 \quad 1.700730 \quad 0.153335$

C $\quad-2.526199 \quad 3.665108 \quad-0.986939$

H $\quad-2.369334 \quad 2.211039 \quad-2.620577$ 


\begin{tabular}{lrrr}
$\mathrm{C}$ & -3.897093 & 2.849566 & 0.825107 \\
$\mathrm{H}$ & -4.622955 & 0.936734 & 0.619145 \\
$\mathrm{C}$ & -3.138230 & 3.844244 & 0.288090 \\
$\mathrm{~N}$ & -1.691204 & 4.582041 & -1.497027 \\
$\mathrm{H}$ & -4.322263 & 2.915302 & 1.817921 \\
$\mathrm{H}$ & -2.933007 & 4.714550 & 0.892050 \\
$\mathrm{C}$ & -0.951463 & 4.284474 & -2.717317 \\
$\mathrm{C}$ & -1.222578 & 5.679548 & -0.653078 \\
$\mathrm{H}$ & -1.637707 & 4.058253 & -3.539105 \\
$\mathrm{H}$ & -0.374637 & 5.163827 & -2.999968 \\
$\mathrm{H}$ & -0.264264 & 3.448850 & -2.543115 \\
$\mathrm{H}$ & -0.750602 & 5.284723 & 0.253835 \\
$\mathrm{H}$ & -0.488472 & 6.257882 & -1.211330 \\
$\mathrm{H}$ & -2.051075 & 6.346541 & -0.391960 \\
$\mathrm{O}$ & -5.271268 & -0.530466 & 1.497660 \\
$\mathrm{~S}$ & -4.352062 & -1.669254 & 1.530335 \\
$\mathrm{H}$ & -3.400203 & -1.793048 & 2.615442 \\
$\mathrm{H}$ & -1.630552 & 0.569522 & 1.225623 \\
$\mathrm{H}$ & -3.611517 & -1.671894 & 0.129538 \\
$\mathrm{C}$ & -5.335119 & -3.147257 & 1.433609 \\
$\mathrm{H}$ & -5.942924 & -3.188113 & 2.338158 \\
$\mathrm{H}$ & -5.965471 & -3.089084 & 0.546752 \\
$\mathrm{H}$ & -4.660613 & -4.002355 & 1.385298 \\
$\mathrm{H}$ & -2.618473 & -1.839463 & 0.192148 \\
$\mathrm{O}$ & -1.034899 & 1.205139 & 0.720874 \\
$\mathrm{H}$ & -3.766715 & 0.526880 & -1.509243 \\
$\mathrm{H}$ & -1.002043 & 0.272589 & -0.532562 \\
$\mathrm{O}$ & 0.882798 & -1.306293 & -0.544543 \\
$\mathrm{C}$ & -1.971477 & -1.085943 & -1.423555 \\
$\mathrm{H}$ & 2.081857 & -1.939155 & -2.108856 \\
\hline & 0.021233 & 2.271433 & 0.833648 \\
$\mathrm{H}$ & -1.306976 & 3.418758 & 1.644068 \\
\hline & -3.673656 & -1.946236 \\
\hline & -2.717333 & -2.741103 \\
\hline
\end{tabular}




$\begin{array}{rrrr}\mathrm{O} & 0.549791 & 2.648166 & -0.498249 \\ \mathrm{C} & 1.881286 & 0.220725 & -2.207770 \\ \mathrm{H} & 1.743001 & 1.071204 & -1.535166 \\ \mathrm{H} & 2.792832 & 0.357139 & -2.793102 \\ \mathrm{H} & 1.023749 & 0.191807 & -2.884357 \\ \mathrm{C} & 5.524781 & -0.866804 & -0.481748 \\ \mathrm{H} & 5.625760 & -1.764637 & 0.133557 \\ \mathrm{C} & 6.671770 & -0.742022 & -1.471504 \\ \mathrm{H} & 6.686016 & -1.611234 & -2.132746 \\ \mathrm{H} & 6.556457 & 0.161568 & -2.075470 \\ \mathrm{H} & 7.621223 & -0.694235 & -0.932949 \\ \mathrm{C} & 6.278057 & 1.219641 & 2.483712 \\ \mathrm{H} & 6.232591 & 2.187601 & 1.979258 \\ \mathrm{H} & 7.242508 & 1.112489 & 2.982629 \\ \mathrm{O} & 4.318755 & -0.943248 & -1.240505 \\ \mathrm{O} & 6.275321 & 0.175276 & 1.487616 \\ \mathrm{C} & 3.197980 & -1.124682 & -0.521818 \\ \mathrm{C} & 5.459888 & 0.346203 & 0.444968 \\ \mathrm{O} & 3.192932 & -1.305175 & 0.670617 \\ \mathrm{O} & 4.795576 & 1.331809 & 0.233908 \\ \mathrm{C} & 5.121486 & 1.040674 & 3.449011 \\ \mathrm{H} & 4.165970 & 1.121266 & 2.925918 \\ \mathrm{H} & 5.172292 & 0.064326 & 3.937566 \\ \mathrm{H} & 5.161647 & 1.818432 & 4.217609\end{array}$

TS P2
C $\quad 0.156738 \quad-4.031379 \quad-0.739707$
$\begin{array}{lllll}\mathrm{O} & -1.078932 & -2.077003 & 0.142512\end{array}$
C $\quad-0.569378-2.239443 \quad-2.240654$
$\begin{array}{llll}\text { C } & -0.480411 & -3.341618 & 0.466519\end{array}$
$\begin{array}{llll}\text { O } & 0.745837 & -5.072155 & -0.586625\end{array}$
H $\quad-0.006637 \quad-1.827859 \quad-3.083983$
C $\quad-0.418770 \quad-1.260472 \quad-1.070940$
$\begin{array}{llll}\mathrm{O} & -1.044344 & -0.158584 & -1.195277\end{array}$
$\begin{array}{llll}\text { C } & -1.498700 & -4.255096 & 1.132597\end{array}$
H $\quad-0.994150 \quad-5.173797 \quad 1.434545$
$\begin{array}{llll}H & -1.922918 & -3.759593 & 2.008434\end{array}$
H $\quad-2.306726 \quad-4.509465 \quad 0.439862$ 

H $\quad 0.343689 \quad-3.123704 \quad 1.150306$
$\begin{array}{llll}\text { C } & -3.599067 & 1.395564 & -0.902171\end{array}$
C $\quad-2.922869 \quad 2.412479-1.517005$
$\begin{array}{llll}N & -4.096580 & 1.537675 & 0.339678\end{array}$
$\begin{array}{llll}\text { C } & -2.691895 & 3.632149 & -0.824297\end{array}$
H $\quad-2.488973 \quad 2.220458 \quad-2.486941$
$\begin{array}{llll}\text { C } & -3.924452 & 2.685785 & 1.023191\end{array}$
H $\quad-4.552886 \quad 0.7205290 .792786$
$\begin{array}{llll}\text { C } & -3.253154 & 3.742762 & 0.480045\end{array}$
$\begin{array}{llll}N & -1.956653 & 4.623442 & -1.359566\end{array}$
$\begin{array}{llll}H & -4.326542 & 2.706725 & 2.028001\end{array}$
$\mathrm{H} \quad-3.097942 \quad 4.621160 \quad 1.088353$
C $\quad-1.285279 \quad 4.418232-2.636560$
C $\quad-1.578505 \quad 5.770809 \quad-0.541249$
$\mathrm{H} \quad-2.010460 \quad 4.178213 \quad-3.420661$
$\begin{array}{llll}H & -0.781606 & 5.341025 & -2.920457\end{array}$
$\begin{array}{llll}H & -0.537690 & 3.621856 & -2.552323\end{array}$
$\begin{array}{llll}\mathrm{H} & -1.022210 & 5.445554 & 0.344686\end{array}$
H $\quad-0.941479 \quad 6.426838 \quad-1.132130$
$\begin{array}{llll}H & -2.463192 & 6.342061 & -0.239755\end{array}$
$\begin{array}{llll}\text { O } & -5.210762 & -0.675061 & 1.437372\end{array}$
S $\quad-4.341982 \quad-1.877353 \quad 1.431535$
$\begin{array}{lllll}\mathrm{O} & -3.528235 & -2.084093 & 2.627786\end{array}$
$\begin{array}{llll}\mathrm{O} & -3.517473 & -1.907562 & 0.154548\end{array}$
$\begin{array}{llll}\text { C } & -5.448989 & -3.266001 & 1.270967\end{array}$
H $\quad-6.104002 \quad-3.269665 \quad 2.142538$
$\begin{array}{llll}\mathrm{H} & -6.026747 & -3.148207 & 0.354420\end{array}$
H $\quad-4.849675 \quad-4.176553 \quad 1.240332$
$\begin{array}{llll}H & -2.154335 & -2.006180 & 0.183406\end{array}$
$\begin{array}{llll}\text { O } & -0.891309 & 1.360879 & 0.721932\end{array}$
$\begin{array}{llll}\mathrm{H} & -3.721637 & 0.417317 & -1.349121\end{array}$
H $\quad-0.889898 \quad 0.704895 \quad-0.138926$
$\begin{array}{lllll}\text { O } & 0.880779 & -1.210979 & -0.542325\end{array}$
C $\quad 1.981331-1.092343 \quad-1.427264$
H $\quad 2.024329-1.962818-2.098066$
S $\quad 0.236174 \quad 2.436109 \quad 0.726182$
$\begin{array}{llll}\mathrm{O} & -0.197352 & 3.544165 & 1.573451\end{array}$
$\begin{array}{llll}\text { O } & 0.083538 & -3.493004 & -1.967538\end{array}$
$\begin{array}{llll}\text { C } & -2.004799 & -2.469178 & -2.673278\end{array}$
H $\quad-2.457012 \quad-1.514662 \quad-2.949037$ 


$\begin{array}{lrrr}\text { H } & -2.017713 & -3.137947 & -3.536840 \\ \text { H } & -2.603884 & -2.909879 & -1.873286 \\ \text { C } & 1.593520 & 1.606842 & 1.523436 \\ \text { H } & 1.745059 & 0.640423 & 1.041741 \\ \text { H } & 2.479611 & 2.233614 & 1.415028 \\ \text { H } & 1.329470 & 1.468541 & 2.572067 \\ \text { O } & 0.629957 & 2.756696 & -0.648275 \\ \text { C } & 1.985218 & 0.202235 & -2.237959 \\ \text { H } & 1.989393 & 1.076736 & -1.584880 \\ \text { H } & 2.866988 & 0.225693 & -2.881040 \\ \text { H } & 1.087718 & 0.260532 & -2.857839 \\ \text { C } & 5.537569 & -1.005307 & -0.482976 \\ \text { H } & 5.608594 & -1.885780 & 0.161118 \\ \text { C } & 6.692596 & -0.949325 & -1.470149 \\ \text { H } & 6.679793 & -1.839378 & -2.102885 \\ \text { H } & 6.610398 & -0.062948 & -2.104334 \\ \text { H } & 7.640662 & -0.916107 & -0.928553 \\ \text { C } & 6.425115 & 1.178332 & 2.373676 \\ \text { H } & 6.357896 & 2.129472 & 1.840553 \\ \text { H } & 7.412819 & 1.083530 & 2.827151 \\ \text { O } & 4.333071 & -1.066020 & -1.243806 \\ \text { O } & 6.371057 & 0.101993 & 1.413559 \\ \text { C } & 3.204458 & -1.191678 & -0.526417 \\ \text { C } & 5.506094 & 0.236111 & 0.407690 \\ \text { O } & 3.188121 & -1.357086 & 0.668391 \\ \text { O } & 4.821753 & 1.210620 & 0.202842 \\ \text { C } & 5.318871 & 1.035866 & 3.401873 \\ \text { H } & 4.338006 & 1.106248 & 2.926280 \\ \text { H } & 5.390068 & 0.074779 & 3.917213 \\ \text { H } & 5.402255 & 1.836039 & 4.143400\end{array}$

Complex P2

$\begin{array}{llll}\text { C } & 2.128072 & -2.413326 & -0.892477 \\ \text { O } & 0.118461 & -1.585051 & 0.188060 \\ \text { C } & 0.556066 & -1.332625 & -2.545904 \\ \text { C } & 1.162600 & -2.508828 & 0.288289 \\ \text { O } & 3.240590 & -2.877716 & -0.821394 \\ \text { H } & 0.825933 & -0.958173 & -3.543392\end{array}$ 


\begin{tabular}{lrrr}
$\mathrm{C}$ & -0.038073 & -0.134123 & -1.808069 \\
$\mathrm{O}$ & -1.240864 & 0.000392 & -1.673748 \\
$\mathrm{C}$ & 0.711678 & -3.958690 & 0.474078 \\
$\mathrm{H}$ & 1.586452 & -4.602624 & 0.587748 \\
$\mathrm{H}$ & 0.088485 & -4.026467 & 1.369419 \\
$\mathrm{H}$ & 0.125341 & -4.303835 & -0.383020 \\
$\mathrm{H}$ & 1.793648 & -2.230142 & 1.141711 \\
$\mathrm{C}$ & -4.265317 & -0.298455 & -0.670230 \\
$\mathrm{C}$ & -4.269248 & 0.970914 & -1.181993 \\
$\mathrm{~N}$ & -4.653829 & -0.543743 & 0.593457 \\
$\mathrm{C}$ & -4.624158 & 2.068161 & -0.351694 \\
$\mathrm{H}$ & -3.896020 & 1.115578 & -2.185119 \\
$\mathrm{C}$ & -5.052551 & 0.459987 & 1.395230 \\
$\mathrm{H}$ & -4.564001 & -1.524578 & 0.967386 \\
$\mathrm{C}$ & -5.068187 & 1.757134 & 0.963418 \\
$\mathrm{~N}$ & -4.509661 & 3.342664 & -0.774344 \\
$\mathrm{H}$ & -5.331516 & 0.180360 & 2.403455 \\
$\mathrm{H}$ & -1.579933 & 0.810786 & -0.215259 \\
$\mathrm{H}$ & -5.347102 & 2.531024 & 1.663278 \\
$\mathrm{C}$ & -3.898326 & 3.625824 & -2.066640 \\
$\mathrm{C}$ & -4.735409 & 4.441629 & 0.158129 \\
$\mathrm{H}$ & -4.462551 & 3.153300 & -2.877166 \\
$\mathrm{H}$ & -3.912158 & 4.701718 & -2.233220 \\
$\mathrm{H}$ & -2.855859 & 3.288281 & -2.086827 \\
$\mathrm{H}$ & -4.041414 & 4.382508 & 1.003909 \\
$\mathrm{H}$ & -4.568227 & 5.382955 & -0.362802 \\
$\mathrm{H}$ & -5.768663 & 4.437116 & 0.521437 \\
$\mathrm{O}$ & -4.326176 & -3.032496 & 1.461964 \\
$\mathrm{~S}$ & -2.942566 & -3.532894 & 1.190876 \\
$\mathrm{O}$ & -2.081570 & -3.617387 & 2.373910 \\
\hline & -2.327715 & -2.774468 & 0.054572 \\
\hline & -3.169221 & -5.200263 & 0.587661 \\
\hline
\end{tabular}




\begin{tabular}{|c|c|c|c|}
\hline & 2.309602 & 0.573606 & \\
\hline & -1.254288 & 2.606394 & 0.955269 \\
\hline & -2.138477 & 3.216125 & $1.93760 \Omega$ \\
\hline & 1.829981 & -1.740265 & -2.039397 \\
\hline & -0.436082 & -2.466738 & $-2.73243 c$ \\
\hline & -1.242453 & -2.134786 & -3.39013 \\
\hline & 0.078140 & -3.305433 & -3.2 \\
\hline & -0.888269 & -2.782523 & -1.79231 \\
\hline & 0.306341 & 2.245390 & 1.7 \\
\hline & 0.894943 & 1.610 & \\
\hline & 0.805645 & 3.197006 & 1.908873 \\
\hline & & & \\
\hline & -1.037627 & 3.291983 & 3805 \\
\hline & 2.315880 & 2.566488 & -1.90755 \\
\hline & 2.059351 & 3.046739 & -0.960966 \\
\hline & 3.361731 & 2.777643 & -2.141740 \\
\hline & 1.672814 & 2.975681 & -2.689077 \\
\hline & 5.209068 & -0.320728 & \\
\hline & 4.759087 & -1.0 & \\
\hline & 6.294373 & 2406 & -1 \\
\hline & 5.854755 & -1.784478 & -1.8 \\
\hline & 6.729992 & -0.234598 & -1.933235 \\
\hline & 7.079502 & -1.358363 & -0.596109 \\
\hline & 7.033394 & 1.368842 & \\
\hline & 7.349416 & 2.243310 & 1.772408 \\
\hline & 7.908459 & 0.878036 & 2.774607 \\
\hline & 4.209085 & 0.170961 & -1.290416 \\
\hline & 6.488887 & 0.389515 & \\
\hline & 3.033176 & 0.478814 & -0.748662 \\
\hline & 5.726091 & 0.843935 & 0.441409 \\
\hline & 2.749305 & 0.345713 & 0.418622 \\
\hline & 5.493587 & 2.009392 & 0.220764 \\
\hline & 6.020985 & 1.740262 & 3.412794 \\
\hline & 5.149475 & 2.224292 & 2.96625 \\
\hline & 5.692914 & 0.853056 & $3.96053 \varepsilon$ \\
\hline & 6.476820 & 2.436979 & 4.12 \\
\hline
\end{tabular}

